\title{
Error estimates for semi-discrete dendritic growth
}

\author{
ANDREAS VEESER \\ Institut für Angewandte Mathematik, Universität Freiburg, \\ Hermann-Herder-Str. 10, D-79104 Freiburg, Germany
}

[Received 22 December 1998 and in revised form 17 March 1999]

\begin{abstract}
Semi-discrete approximations to a mathematical model for two-dimensional dendritic growth are analysed. The model is a Stefan problem with interfacial structure. The semi-discrete problem uses a parametrization for the free boundary and finite elements in space. The main results are a priori error estimates for the temperature field and the parametrization of the free boundary. The optimality of their order is discussed. Further error estimates concern approximations to relevant geometric (e.g. curvature) and measuring quantities.
\end{abstract}

\section{Introduction}

Various methods for the numerical simulation of dendritic growth have been proposed and tested (see for example $[8,19,22,31]$ and the references therein). But their numerical analysis is not investigated well. This article concerns the numerical analysis of a finite element method that has the same structure as the approach that was used in $[1,22]$. Its goal is to prove that spatially semidiscrete solutions converge to a solution of the underlying mathematical model when the mesh size decreases to 0 .

The underlying model is a well-known generalization of the classical Stefan problem $[6,15,18$, $20,30]$. Let $\Omega \subset \mathbb{R}^{2}$ denote a region occupied by a pure material that may be in solid or liquid phase. The region $\Omega$ divides into the solid region $\omega^{s}$, the liquid region $\omega^{l}$ and the one-dimensional interface $\gamma$ that separates $\omega^{s}$ and $\omega^{l}$. The partition $p=\left(\omega^{s}, \gamma, \omega^{l}\right)$ of the region $\Omega$ depends on time $t$. We associate the normal $n$, the normal velocity $v$ and the curvature $k$ of the interface $\gamma$ with the partition $p$. The normal $n$ points into the liquid region $\omega^{l}$ and the curvature $k$ is positive, if the solid region $\omega^{s}$ is convex. Finally, [[. $\left.]\right]_{s}^{l}$ denotes the jump across the interface $\gamma$ with the sign convention 'liquid'-'solid'. Using this notation the model consists of the following evolution laws for the (scaled) temperature field $\theta=\theta(x, t)$ and phase distribution $p=p(t)$ :

$$
\begin{aligned}
\theta_{t}-\Delta \theta=0 & \text { in } \omega^{s}(t) \cup \omega^{l}(t), \\
v=-\llbracket \frac{\partial \theta}{\partial n} \rrbracket_{s}^{l} & \text { on } \gamma(t), \\
\beta(n) v+a(n) k+\theta=0 & \text { on } \gamma(t),
\end{aligned}
$$

where $\beta$ and $a$ denote material quantities. The function $\beta$, defined on the sphere $\mathbb{S}:=\left\{x \in \mathbb{R}^{2} \mid\right.$ $|x|=1\}$, is called the kinetic coefficient and is involved in the term $\beta(n) v$, which models molecular attachment. The function $a$ is the interface potential. It can be calculated from a smooth surface tension $\phi=\phi(n)$ by the formula $a(n)=D^{2} \phi(n) n^{\perp} \cdot n^{\perp}$ where $D^{2} \phi(n)$ is the Hessian of the positively homogeneous extension of $\phi$ on $\mathbb{R}^{2}$ and ${ }^{\perp}$ denotes the counterclockwise rotation by 
$90^{\circ}$. The last equation in model (1.1), a generalized Gibbs-Thomson law, makes the difference to the classical Stefan problem; it allows for a shift of the interfacial temperature due to kinetic and curvature effects.

We recall some features of (1.1) about its usefulness as a model for dendritic growth. Model (1.1) incorporates heat transport, latent heat, anisotropic surface tension and molecular attachment effects. It is widely believed that a coupling of these four effects should explain dendritic growth (see [15] and the morphological stability analyses [7,27] as well as the references therein). Moreover, model (1.1) is an 'approximation' of a model that is consistent with thermodynamics [17].

In the sequel we restrict ourselves to the case that the material quantities satisfy

$$
\beta_{*}:=\inf _{n \in \mathbb{S}} \beta(n)>0 \quad \text { and } \quad a_{*}:=\inf _{n \in \mathbb{S}} a(n)>0 .
$$

If we assume additionally that the material is isotropic, i.e. $\beta$ and $a$ are constant functions, then two results concerning the well-posedness of model (1.1) are known. Model (1.1), together with appropriate initial and boundary conditions, admits a unique classical solution on a short time interval [9]. There are global weak solutions [26].

In Section 2 we develop a semi-discrete method for model (1.1). The method cannot handle 'geometric singularities' such as merging or separation of phase regions. Nevertheless it can be applied to simulate important experiments in dendritic growth. The part of the method that concerns the discretization of the generalized Gibbs-Thomson law, a driven motion by anisotropic curvature, uses the same approach as in [11] and [12]. The 'derivation' of the method is presented in two steps. The first step is a reformulation of (1.1) introducing a parametrization $g(\cdot, t)$ of the free boundary $\gamma(t)$. A system for the parametrizations $g=g(u, t)$, which is parabolic thanks to (1.2), replaces the generalized Gibbs-Thomson law. The second step is an application of the standard procedure to get a finite element method from a classical formulation. The unknowns of the resulting semidiscrete problem are the approximations $\Theta$ and $G$ to the temperature field $\theta$ and the parametrization $g$ respectively.

In Section 3 error estimates for the described method are presented. They cover the case that the free boundary $\gamma$ is parametrized over the sphere $\mathbb{S}$ and does not touch the boundary $\partial \Omega$. The most important ones are

$$
\left.\begin{array}{l}
\sup _{(0, T)}\|\Theta-\theta\|_{L_{2}(\Omega)}^{2}, \quad h \int_{0}^{T}\|\nabla(\Theta-\theta)\|_{L_{2}(\Omega)}^{2}, \\
\sup _{(0, T)}\|G-g\|_{H^{1}\left(\mathbb{S}, \mathbb{R}^{2}\right)}^{2}, \\
\int_{0}^{T}\left\|G_{t}-g_{t}\right\|_{L_{2}\left(\mathbb{S}, \mathbb{R}^{2}\right)}^{2}
\end{array}\right\} \leqslant C h^{2},
$$

where $h \in\left(0, h^{*}\right]$ is the mesh size. Here the constants $h^{*}>0$ and $C \geqslant 0$ depend on reasonable regularity (see Remark 2.1) and geometric properties of the continuous solution $(\theta, g)$. The $H^{1}$ estimate for the parametrization of the free boundary implies error estimates for the phase regions $\omega^{l}$ and $\omega^{s}$. Further estimates concern approximations to the normal $n$, the normal velocity $v$ and the curvature $k$. From these follow more estimates for approximations to the velocity and radius of a primary dendrite tip under some additional assumptions. The velocity and the radius are measured in order to investigate the steady-state propagation of a primary tip. We summarize these results by concluding that the described method is reliable and, moreover, that it can be applied in principle to compare 'dendrites of model (1.1)' with real ones. 
Section 4 contains the proof of these results. The main difficulties in the establishment of the estimates (1.3) are an appropriate control of the traces occurring in coupling terms and the limited regularity of the temperature field $\theta$. An interaction of these aspects suggests that the estimates (1.3) are of optimal order.

\section{Reformulation and semi-discrete problem}

We employ parametrizations (here: continuous mappings into $\mathbb{R}^{2}$ ) of the free boundary to rewrite model (1.1). For simplicity, the domain of definition of these parametrizations is the sphere $\mathbb{S}$. We identify derivatives of mappings with their representations with respect to 'canonical' bases in the involved tangential spaces. In the context of $\mathbb{R}^{n}$ 'canonical' has the usual meaning, whereas, for example, in the case of the tangential space $T \mathbb{S}_{u}$ at $u \in \mathbb{S}$ it indicates the choice $u^{\perp}$. In order to recover the phase regions from the parametrizations we use the following 'oriented distance function'. Let $f: \mathbb{S} \rightarrow \mathbb{R}^{2}$ be an injective parametrization and define $\operatorname{sdist}(f, \cdot): \mathbb{R}^{2} \rightarrow \mathbb{R}$ by

$$
\operatorname{sdist}(f, x):=\operatorname{Or}(f) \operatorname{sdist}(\partial \operatorname{Int}(f), x),
$$

where $\operatorname{Or}(f) \in\{ \pm 1\}$ denotes the orientation of $f, \operatorname{Int}(f)$ is the interior of the Jordan curve $f(\mathbb{S})$ (see for example Section IV.5 in [5]), and $\operatorname{sist}(\partial A, x):=\operatorname{dist}(A, x)-\operatorname{dist}\left(\mathbb{R}^{2} \backslash A, x\right)$ with $\operatorname{dist}(A, x):=$ $\inf _{y \in A}|y-x|$ for $A \subset \mathbb{R}^{2}$.

Let $\Omega \subset \mathbb{R}^{2}$ be a bounded domain, let $T>0$ be fixed and set $Q:=\Omega \times(0, T)$ as well as $\mathbb{S}_{T}:=\mathbb{S} \times(0, T)$. Let $\theta_{0}$ be an extension on $\bar{Q}$ of the initial and boundary temperature, and let $g_{0}$ be an injective parametrization of a Jordan curve (the initial interface) in $\Omega$.

PROBLem 2.1 Find $\theta=\theta(x, t)$ and $g=g(u, t)$ such that

(i) $g \in C^{2,1}\left(\mathbb{S}_{T}, \mathbb{R}^{2}\right)$, and $g(t):=g(\cdot, t)$ is a regular, injective parametrization with $g(\mathbb{S}, t) \subset \Omega$ for every $t \in(0, T)$,

(ii) $\theta$ is of class $C^{2,1}$ in $Q^{s}:=\{(x, t) \in Q \mid \operatorname{sdist}(g(t), x)<0\}$ and in $Q^{l}:=\{(x, t) \in Q \mid$ sdist $(g(t), x)>0\}$, and $\nabla \theta$ exists on $g\left(\mathbb{S}_{T}\right)$ 'from both sides',

(iii) the equations

$$
\begin{aligned}
\theta_{t} & =\Delta \theta & & \text { in } Q^{s} \cup Q^{l}, \\
g_{t} \cdot n & =-\llbracket[\nabla \theta]_{s}^{l}(g) \cdot n & & \text { on } \mathbb{S}_{T}, \\
\beta(n) g_{t} & =\frac{a(n)}{\left|g_{u}\right|^{2}} g_{u u}-\theta(g) n & & \text { on } \mathbb{S}_{T}
\end{aligned}
$$

with $n=-g_{u}^{\perp} /\left|g_{u}\right|$ hold,

(iv) the initial and boundary conditions

$$
\begin{array}{ll}
g(u, 0)=g_{0}(u) & \text { for all } u \in \mathbb{S}, \\
\theta(x, t)=\theta_{0}(x, t) & \text { for all }(x, t) \in[\Omega \times\{0\}] \cup[\partial \Omega \times(0, T)]
\end{array}
$$

are fulfilled. 
Hereafter, $\theta(g)$ stands for the function $(u, t) \mapsto \theta(g(u, t), t)$. The expression $\llbracket \nabla \theta \rrbracket_{S}^{l}(g)$ is interpreted in a similar way. Condition (i) implies that $Q^{s}$ and $Q^{l}$ are open (see Section 20 in [29]). Moreover, it excludes that the interface touches the boundary $\partial \Omega$. The third equation in condition (iii) implies the generalized Gibbs-Thomson law (multiply by the normal $n$ ) and is a 'singular', parabolic system in the sense of Petrovsky [13] thanks to (1.2).

REMARK 2.1 Let us assume that the material is isotropic and that the data is sufficiently smooth and compatible. In [9] Chen and Reitich prove that there exists a $T>0$ such that model (1.1) admits a unique solution $(\theta, p)$ on $[0, T]$, thereby representing the interface $\gamma$ as 'a graph over the initial interface'. We find a solution $(\theta, g)$ of Problem 2.1 by reparametrizing the interface $\gamma$ of $(\theta, p)$ in an appropriate way. This solution $(\theta, g)$ has the regularity

$$
\begin{gathered}
g \in C^{3+\alpha,(3+\alpha) / 2}\left(\overline{\mathbb{S}}_{T}, \mathbb{R}^{2}\right), \quad g_{t} \in L_{2}\left(0, T ; H^{2}\left(\mathbb{S}, \mathbb{R}^{2}\right)\right), \\
\theta \in C^{0}(\bar{Q}),\left.\quad \theta\right|_{Q^{s}} \in C^{2+\alpha,(2+\alpha) / 2}\left(\overline{Q^{s}}\right),\left.\quad \theta\right|_{Q^{l}} \in C^{2+\alpha,(2+\alpha) / 2}\left(\overline{Q^{l}}\right)
\end{gathered}
$$

with $\alpha \in(0,1)$. Moreover, condition (i) is also satisfied for $t=0$ (by assumption) and $t=T$. (See Section 19 in [29].)

In order to state the semi-discrete problem (and for later purposes) we define

$$
\underline{\mathrm{DQ}}(f):=\inf \left\{\frac{\left|f\left(u_{1}\right)-f\left(u_{2}\right)\right|}{d_{\mathbb{S}}\left(u_{1}, u_{2}\right)} \mid u_{1}, u_{2} \in \mathbb{S}, u_{1} \neq u_{2}\right\},
$$

where $f: \mathbb{S} \rightarrow \mathbb{R}^{2}$ is a parametrization and $d_{\mathbb{S}}$ denotes the standard distance on the sphere $\mathbb{S}$. The condition $\mathrm{DQ}(f)>0$ implies that $f$ is injective and that the length element $\left|f_{u}\right|$ is bounded away from zero where it exists.

Let $X$ and $Y$ be finite-dimensional subspaces, e.g. (linear) finite element spaces, of $W_{\infty}^{1}(\Omega)$ and $W_{\infty}^{1}\left(\mathbb{S}, \mathbb{R}^{2}\right)$ respectively, and set $X:=X \cap H^{1}(\Omega)$. Let $\Theta_{0} \in X$ and $G_{0} \in Y$ be approximations to the initial and boundary values $\theta_{0}$ and $g_{0}$ respectively. Furthermore, let $G_{0}$ satisfy $\operatorname{DQ}\left(G_{0}\right)>0$ and $G_{0}(\mathbb{S}) \subset \Omega$.

Problem 2.2 Find $\Theta:[0, T] \rightarrow X$ and $G:[0, T] \rightarrow Y$ such that

(i) $G \in H^{1}(0, T ; Y)$, and $G(\mathbb{S}, t) \subset \Omega$ as well as $\underline{\mathrm{DQ}}(G(t))>0$ for every $t \in(0, T)$,

(ii) $\Theta \in H^{1}(0, T ; X)$,

(iii) the equations

$$
\begin{array}{rlrl}
\int_{\Omega} \Theta_{t} \Phi+\int_{\Omega} \nabla \Theta \cdot \nabla \Phi & =-\int_{\mathbb{S}} G_{t} \cdot G_{u}^{\perp} \Phi(G) & & \forall \Phi \in \dot{X}, \\
\int_{\mathbb{S}} \frac{\beta(N)}{a(N)} G_{t} \cdot \Psi\left|G_{u}\right|^{2}+\int_{\mathbb{S}} G_{u} \cdot \Psi_{u} & =\int_{\mathbb{S}} \frac{\Theta(G)}{a(N)} G_{u}^{\perp} \cdot \Psi\left|G_{u}\right| & \forall \Psi \in Y
\end{array}
$$

with $N=-G_{u}^{\perp} /\left|G_{u}\right|$ hold almost everywhere in $(0, T)$,

(iv) the initial and boundary conditions

$$
G(0)=G_{0}, \quad \Theta(0)=\Theta_{0}, \quad \Theta(t)-\Theta_{0}(t) \in \stackrel{X}{X} \text { for all } t \in(0, T)
$$

are fulfilled. 
The equation for $G$ can be obtained in the following way: let $\psi=\left(\psi_{1}, \psi_{2}\right)$ be a test function, take the scalar product of the system for $g$ and $\psi\left|g_{u}\right|^{2} / a(n)$, integrate over the sphere $\mathbb{S}$, integrate by parts the principal term and then replace the solution space as well as the test space by $Y$. The equation for $\Theta$ can be obtained in a similar way; after integration by parts use the second equation in condition (iii) of the parametric formulation Problem 2.1.

We can rewrite the semi-discrete Problem 2.2 as an initial value problem for a system of ordinary differential equations by choosing bases in $X$ and $Y$. The right-hand side of that system satisfies a locally uniform Lipschitz condition. Consequently, the Theorem of Picard-Lindelöf implies the following local existence and uniqueness result.

Lemma 2.1 Assume that $\operatorname{DQ}\left(G_{0}\right)>0, G_{0}(\mathbb{S}) \subset \Omega$, and $\Theta_{0} \in H^{1}(0, T ; X)$. Then there exists a $t^{*} \in(0, T]$ such that Problem 2.2 admits a unique solution on $\left[0, t^{*}\right]$. Moreover, $G \in C^{1}\left(\left[0, t^{*}\right], Y\right)$.

\section{Results}

The numerical analysis of Problem 2.2 relies on the following assumptions. Let us start with the assumptions on data $\Omega, \beta, a, \theta_{0}$ and $g_{0}$. Let $\Omega$ be a bounded, simply-connected domain with Lipschitz boundary and a constant $c_{\text {ell }} \geqslant 0$ such that

$$
\varphi \in \stackrel{\circ}{H}^{1}(\Omega),-\Delta \varphi \in L_{2}(\Omega) \quad \Longrightarrow \quad\|\varphi\|_{H^{2}(\Omega)} \leqslant c_{\mathrm{ell}}\|\Delta \varphi\|_{L_{2}(\Omega)}
$$

is satisfied. Regarding the material quantities $\beta$ and $a$ assume $\beta, a \in W_{\infty}^{1}(\mathbb{S})$ in addition to (1.2). Let the initial and boundary temperature $\theta_{0}=\theta_{0}^{1}+\theta_{0}^{2}$ be split into a time-dependent part $\theta_{0}^{1}$ and a time-independent part $\theta_{0}^{2}$ such that

$$
\begin{gathered}
\theta_{0}^{1} \in W_{\infty}^{1}(Q) \cap L_{2}\left(0, T ; H^{2}(\Omega)\right), \\
\theta_{0}^{2} \in W_{\infty}^{1}(\Omega) \cap \stackrel{H}{ }^{1}(\Omega),\left.\quad \theta_{0}^{2}\right|_{\omega_{0}^{s}} \in H^{2}\left(\omega_{0}^{s}\right),\left.\quad \theta_{0}^{2}\right|_{\omega_{0}^{l}} \in H^{2}\left(\omega_{0}^{l}\right),
\end{gathered}
$$

where $\omega_{0}^{s}=\left\{x \in \Omega \mid \operatorname{sdist}\left(g_{0}, x\right)<0\right\}$ and $\omega_{0}^{l}=\left\{x \in \Omega \mid \operatorname{sdist}\left(g_{0}, x\right)>0\right\}$. This splitting takes into account that the compatibility condition on the free boundary used in Remark 2.1 may imply a jump of $\nabla \theta_{0}$ across the initial interface.

Let us continue with the assumptions on the discrete spaces $X$ and $Y$. Let $h>0$ indicate the discretization parameter, and let $c_{\text {inv }}$ and $c_{\text {aprx }}$ be two positive constants, which do not depend on $h$. Let $X \subset W_{\infty}^{1}(\Omega)$ and $Y=Z^{2}, Z \subset W_{\infty}^{1}(\mathbb{S})$ fulfill the inverse assumptions

$$
\begin{array}{ll}
\|\nabla \Phi\|_{L_{2}(\Omega)} \leqslant c_{\text {inv }} h^{-1}\|\Phi\|_{L_{2}(\Omega)} & \forall \Phi \in X, \\
\left\|\Xi_{u}\right\|_{L_{\infty}(\mathbb{S})} \leqslant c_{\text {inv }} h^{-1 / 2}\left\|\Xi_{u}\right\|_{L_{2}(\mathbb{S})} & \forall \Xi \in Z .
\end{array}
$$

Furthermore, assume that there exist linear interpolation operators $I: H^{1}(\Omega) \rightarrow X$ and $J$ : $H^{1}(\mathbb{S}) \rightarrow Z$ such that

$$
\begin{gathered}
\|I \varphi-\varphi\|_{L_{2}(\Omega)}+h\|\nabla(I \varphi-\varphi)\|_{L_{2}(\Omega)} \leqslant c_{\text {aprx }} h^{m}\|\varphi\|_{H^{m}(\Omega)}, \\
\varphi \in \stackrel{\circ}{ }^{1}(\Omega) \Longrightarrow I \varphi \in \dot{X} \\
\|J \xi-\xi\|_{L_{p}(\mathbb{S})}+h\left\|(J \xi-\xi)_{u}\right\|_{L_{p}(\mathbb{S})} \leqslant c_{\text {aprx }} h^{m}\|\xi\|_{W_{p}^{m}(\mathbb{S})}
\end{gathered}
$$


for $p=2, \infty, m=1,2$ and every $\varphi \in H^{m}(\Omega)$ as well as every $\xi \in W_{p}^{m}(\mathbb{S})$. In addition, assume that there exists a partition of $\Omega$ into Lipschitz domains such that all $\Phi \in X$ are piecewise smooth on this partition. The assumptions on $X$ are satisfied, if we choose $X$ from a family of linear (or higher order) finite element spaces that is given by a quasi-uniform and regular family of triangulations over a polygonal $\Omega$ (see [10] and [25] for the choice of $I$ ). A similar statement holds for the choice of $Y$.

We choose the approximations $G_{0}$ and $\Theta_{0}$ of the initial and boundary values $g_{0}$ and $\theta_{0}$ in the following way: let

$$
G_{0}=J g_{0} \quad \text { and } \quad \Theta_{0}=P \theta_{0}^{1}+R \theta_{0}^{2},
$$

where $J$ acts on each component, $P$ denotes the orthogonal projection $L_{2}(\Omega) \rightarrow X$, and $R$ is the Riesz projection onto $\stackrel{X}{X}$.

Finally, assume that the continuous solution $(\theta, g)$ of Problem 2.1 satisfies

$$
\begin{gathered}
g \in C^{2,1}\left(\overline{\mathbb{S}}_{T}, \mathbb{R}^{2}\right), \quad g_{t} \in C^{1,0}\left(\overline{\mathbb{S}}_{T}, \mathbb{R}^{2}\right), \quad g_{u}, g_{t} \in L_{2}\left(0, T ; H^{2}\left(\mathbb{S}, \mathbb{R}^{2}\right)\right), \\
\theta \in W_{\infty}^{1}(Q), \sup _{t \in(0, T)}\left(\left\|\left.\theta\right|_{Q^{s}}(t)\right\|_{H^{2}\left(\omega^{s}(t)\right)}+\left\|\left.\theta\right|_{Q^{l}}(t)\right\|_{H^{2}\left(\omega^{l}(t)\right)}\right)<\infty
\end{gathered}
$$

with $\omega^{s}(t)=Q^{s} \cap[\Omega \times\{t\}]$ and $\omega^{l}(t)=Q^{l} \cap[\Omega \times\{t\}]$. These regularity assumptions are reasonable; compare with Remark 2.1.

Now we state the results. Their proofs are contained in Section 4.

THEOREM 3.1 Suppose that assumptions (3.1)-(3.8) are satisfied and that the solution $(\theta, g)$ of Problem 2.1 fulfills

$$
\inf _{t \in(0, T)} \mathrm{DQ}(g(t))>0 \text { and } \inf _{t \in(0, T)} \operatorname{dist}(g(\mathbb{S}, t), \partial \Omega)>0 .
$$

Then there exist $h^{*}>0$ and $C \geqslant 0$, depending on given data $\Omega, T, \beta, a, \theta_{0}^{1}, \theta_{0}^{2}, g_{0}$, 'discretization constants' $c_{\text {inv }}, c_{\text {aprx }}$, and the continuous solution $(\theta, g)$, such that for all $h \in\left(0, h^{*}\right]$

(i) there exists a unique solution $(\Theta, G)$ on $[0, T]$ of Problem 2.2,

(ii) the following error estimates for the temperature field and the parametrization of the free boundary hold:

$$
\left.\begin{array}{c}
\sup _{(0, T)}\|\Theta-\theta\|_{L_{2}(\Omega)}^{2}, \quad h \int_{0}^{T}\|\nabla(\Theta-\theta)\|_{L_{2}(\Omega)}^{2}, \\
\int_{0}^{T}\|\Theta(G)-\theta(g)\|_{L_{2}(\mathbb{S})}^{2}, \\
\sup _{(0, T)}\|G-g\|_{H^{1}\left(\mathbb{S}, \mathbb{R}^{2}\right)}^{2}, \quad \int_{0}^{T}\left\|G_{t}-g_{t}\right\|_{L_{2}\left(\mathbb{S}, \mathbb{R}^{2}\right)}^{2}
\end{array}\right\} \leqslant C h^{2} .
$$

REMARK 3.1 If there is a geometric singularity (i.e. a topological change of the interface) at time $T_{\text {sing }}$, we have DQ $(g(T)) \downarrow 0$ when $T \uparrow T_{\text {sing. This implies }}$

$$
h^{*} \downarrow 0 \quad \text { and } \quad C \uparrow \infty \quad \text { as } \quad T \uparrow T_{\text {sing }} .
$$


The error estimates for the free boundary in Theorem 3.1 are rather strong. An illustration of this fact are the following consequences. The first one concerns the phase distribution $p=\left(\omega^{s}, \gamma, \omega^{l}\right)$. Let $\delta$ denote the Hausdorff distance between (arbitrary) sets in $\left(\mathbb{R}^{2},|\cdot|\right)$, and define the discrete phase distribution by $\Gamma(t):=G(\mathbb{S}, t), \Omega^{s}(t):=\{x \in \Omega \mid$ sdist $(G(t), x)<0\}$, and $\Omega^{l}(t):=\{x \in$ $\Omega \mid \operatorname{sdist}(G(t), x)>0\}$ for $t \in[0, T]$.

COROLlary 3.1 Suppose that the assumptions of Theorem 3.1 hold and choose $h^{*}>0$ and $C$ like therein. Then the error of the phase distribution is controlled in the following way: for all $h \in\left(0, h^{*}\right]$

$$
\sup _{(0, T)} \delta\left(\omega^{s}, \Omega^{s}\right), \sup _{(0, T)} \delta(\gamma, \Gamma), \sup _{(0, T)} \delta\left(\omega^{l}, \Omega^{l}\right) \leqslant C h .
$$

A second consequence of the error estimates in Theorem 3.1 concerns the approximation of the geometric quantities $n, v, k$ that appear in model (1.1). In the sequel we assume that $v$ and $k$ (like $n$ ) are defined on the sphere $\mathbb{S}$. Recall $N=-G_{u}^{\perp} /\left|G_{u}\right|$ and choose the approximations of the normal velocity $v$ and curvature $k$ in the following way:

$$
V:=G_{t} \cdot N, \quad K:=-\frac{1}{a(N)}(\beta(N) V+\Theta(G)) .
$$

COROLlary 3.2 Suppose that the assumptions of Theorem 3.1 hold. Then the quantities $N, V, K$ approximate $n, v, k$ respectively, more precisely: there exists a constant $C \geqslant 0$ such that for all $h \in\left(0, h^{*}\right]$, we have

$$
\sup _{(0, T)}\|N-n\|_{L_{2}\left(\mathbb{S}, \mathbb{R}^{2}\right)}^{2}, \int_{0}^{T}\|V-v\|_{L_{2}(\mathbb{S})}^{2}, \int_{0}^{T}\|K-k\|_{L_{2}(\mathbb{S})}^{2} \leqslant C h^{2} .
$$

Corollary 3.2 allows to approximate measuring quantities in dendritic growth experiments, namely the velocity and curvature (= inverse of the radius) of a primary tip. Let $g_{\text {tip }}:(0, T) \rightarrow \mathbb{R}^{2}$ with $g_{\text {tip }}(t) \in \gamma(t)$ be the position vector of a primary tip, and let $G_{\text {tip }}:(0, T) \rightarrow \mathbb{R}^{2}$ with $G_{\text {tip }}(t) \in \Gamma(t)$ some approximation of $g_{\text {tip }}$. Let $Q$ indicate the orthogonal projection $L_{2}(\mathbb{S}) \rightarrow Z$. Define

$$
v_{\text {tip }}(t):=v\left(g^{-1}\left(g_{\text {tip }}(t), t\right), t\right) \quad \text { and } \quad V_{\text {tip }}(t):=Q V\left(G^{-1}\left(G_{\text {tip }}(t), t\right), t\right)
$$

for $t \in[0, T]$, where $g^{-1}(\cdot, t)$ and $G^{-1}(\cdot, t)$ denote the inverse of $g(\cdot, t)$ and $G(\cdot, t)$ respectively. Define $k_{\text {tip }}$ and $K_{\text {tip }}$ in an analogous way. The approximation of $v_{\text {tip }}$ and $k_{\text {tip }}$ by $V_{\text {tip }}$ and $K_{\text {tip }}$ respectively is established in two steps. The first step is a reduction to the task that $G_{\text {tip }}$ approximates $g_{\text {tip }}$ in an appropriate way. The second step establishes such an approximation in a special situation.

COROLlary 3.3 Suppose that the assumptions of Theorem 3.1 hold. Then there exists a constant $C \geqslant 0$ such that for all $h \in\left(0, h^{*}\right]$

$$
\left.\begin{array}{c}
\left\|V_{\text {tip }}-v_{\text {tip }}\right\|_{L_{2}(0, T)}, \\
\left\|K_{\text {tip }}-k_{\text {tip }}\right\|_{L_{2}(0, T)}
\end{array}\right\} \leqslant C\left(h^{1 / 2}+\left\|G_{\text {tip }}-g_{\text {tip }}\right\|_{L_{2}\left((0, T), \mathbb{R}^{2}\right)}\right) .
$$


A variant of the practical construction of $G_{\text {tip }}$ in the sequel was used in [22]. The construction relies on the following assumption on the continuous tip: suppose there is a known unit vector $e$ (given by the anisotropy) such that

$$
g_{\text {tip }}(t) \cdot e^{\perp}=0 \quad \text { and } \quad g_{\text {tip }}(t) \cdot e=\sup \{x \cdot e \mid x \in \gamma(t)\}
$$

for all $t \in(0, T)$.

LEMMA 3.1 Suppose the assumptions of Theorem 3.1 and (3.11). Choose $h^{*}>0$ like in Theorem 3.1 and let $h \in\left(0, h^{*}\right]$. Then there exists a unique measurable mapping $G_{\text {tip }}:[0, T] \rightarrow \mathbb{R}^{2}$ with $G_{\text {tip }}(t) \in \Gamma(t)$,

$$
G_{\text {tip }}(t) \cdot e^{\perp}=0, \quad \text { and } \quad G_{\text {tip }}(t) \cdot e=\sup \left\{x \cdot e \mid x \in \Gamma(t) \text { and } x \cdot e^{\perp}=0\right\}
$$

for all $t \in[0, T]$. Moreover, we have the error estimate

$$
\sup _{(0, T)}\left|G_{\text {tip }}-g_{\text {tip }}\right| \leqslant C h,
$$

where $C \geqslant 0$ does not depend on $h$.

Using Lemma 3.1 in the Inequalities (3.10) we obtain the following error estimates for the velocity and curvature of the tip:

$$
\left\|V_{\text {tip }}-v_{\text {tip }}\right\|_{L_{2}(0, T)},\left\|K_{\text {tip }}-k_{\text {tip }}\right\|_{L_{2}(0, T)} \leqslant C h^{1 / 2},
$$

where $h \in\left(0, h^{*}\right]$ and $C$ does not depend on $h$.

\section{Proof}

Let us divide the proof of the results in the previous section into several steps. At first we establish error estimates for one unknown (or its crucial part) in terms of the other one under some additional assumptions, which are marked with $(+)$. Then we couple these estimates and get rid of the additional assumptions. In doing so, we establish Theorem 3.1 and are able to discuss the optimality of the estimates therein. Finally, we prove Corollaries 3.1-3.3 and Lemma 3.1.

For the sake of simplicity, we assume $\theta_{0}=0$ throughout the proof; the case $\theta_{0} \neq 0$ does not involve new ideas. Suppose that the assumptions of Theorem 3.1 are satisfied. In addition, at the first stage of the proof, let $t^{*} \in(0, T]$ and assume that $(\Theta, G)$ is a solution on $\left[0, t^{*}\right]$ of the semi-discrete Problem 2.2 with $G \in C^{1}\left(\left[0, t^{*}\right], Y\right)$.

\section{Control of the free boundary (Part 1)}

We start with a lemma that implies that the error of the free boundary is controlled by the error of the interfacial temperature. The error of the interfacial temperature will be estimated later on. Set $l_{*}:=\inf _{\mathbb{S}_{T}}\left|g_{u}\right|>0$ and $l^{*}:=\sup _{\mathbb{S}_{T}}\left|g_{u}\right|<\infty$.

LEMMA 4.1 Suppose that

$$
L_{*} \leqslant\left|G_{u}\right| \leqslant L^{*} \quad \text { in } \mathbb{S} \times\left[0, t^{*}\right]
$$


where $L_{*}, L^{*}$ are constants with $0<L_{*} \leqslant L^{*}$. Then there exists a constant $c$, which depends on $L_{*}^{-1}, L^{*}, l_{*}^{-1}, l^{*},\left\|g_{t}\right\|_{C^{1,0}\left(\overline{\mathbb{S}}_{T}, \mathbb{R}^{2}\right)},\left\|g_{0}\right\|_{C^{2}\left(\mathbb{S}, \mathbb{R}^{2}\right)}, \int_{0}^{T}\left\|g_{t}\right\|_{H^{2}\left(\mathbb{S}, \mathbb{R}^{2}\right)}^{2},\|\theta\|_{C^{0}(\bar{Q})}, c_{\text {aprx }}, T$ increasingly and on $\beta, a$, such that for any $t \in\left[0, t^{*}\right]$

$$
\begin{aligned}
\|G(t)-g(t)\|_{H^{1}\left(\mathbb{S}, \mathbb{R}^{2}\right)}^{2}+\int_{0}^{t}\left\|G_{t}-g_{t}\right\|_{L_{2}\left(\mathbb{S}, \mathbb{R}^{2}\right)}^{2} & \\
& \leqslant c\left(h^{2}+\int_{0}^{t}\|G-g\|_{H^{1}\left(\mathbb{S}, \mathbb{R}^{2}\right)}^{2}+\int_{0}^{t}\|\Theta(G)-\theta(g)\|_{L_{2}(\mathbb{S})}^{2}\right) .
\end{aligned}
$$

Proof. Let us test the variational equation for $G$ in Problem 2.2 and the corresponding one for $g$ with $\Psi=\psi=G_{t}-J g_{t}$. Taking their difference, we get

$$
\frac{\beta_{*} L_{*}}{a^{*}}\left\|G_{t}-J g_{t}\right\|_{L_{2}\left(\mathbb{S}, \mathbb{R}^{2}\right)}^{2}+\frac{1}{2} \frac{\mathrm{d}}{\mathrm{d} t}\left\|G_{u}-g_{u}\right\|_{L_{2}\left(\mathbb{S}, \mathbb{R}^{2}\right)}^{2} \leqslant \sum_{k=1}^{4} I_{k},
$$

where

$$
\begin{gathered}
I_{1}:=\int_{\mathbb{S}}\left(\frac{\Theta(G)}{a(N)}\left|G_{u}\right| G_{u}^{\perp}-\frac{\theta(g)}{a(n)}\left|g_{u}\right| g_{u}^{\perp}\right) \cdot\left(G_{t}-J g_{t}\right), \\
I_{2}:=\int_{\mathbb{S}} g_{t} \cdot\left(G_{t}-J g_{t}\right)\left(\frac{\beta(n)}{a(n)}\left|g_{u}\right|^{2}-\frac{\beta(N)}{a(N)}\left|G_{u}\right|^{2}\right), \\
I_{3}:=\int_{\mathbb{S}} \frac{\beta(N)}{a(N)}\left(g_{t}-J g_{t}\right) \cdot\left(G_{t}-J g_{t}\right)\left|G_{u}\right|^{2}, \quad I_{4}:=\int_{\mathbb{S}}\left(G_{u}-g_{u}\right) \cdot\left(\left(J g_{t}\right)_{u}-g_{t u}\right),
\end{gathered}
$$

and $a^{*}:=\sup _{n \in \mathbb{S}} a(n)$. In a straight-forward manner one gets

$$
I_{4} \leqslant \frac{1}{2}\left\|G_{u}-g_{u}\right\|_{L_{2}\left(\mathbb{S}, \mathbb{R}^{2}\right)}^{2}+c\left\|g_{t}\right\|_{H^{2}\left(\mathbb{S}, \mathbb{R}^{2}\right)}^{2} h^{2}
$$

and

$$
I_{3} \leqslant \varepsilon\left\|G_{t}-J g_{t}\right\|_{L_{2}\left(\mathbb{S}, \mathbb{R}^{2}\right)}^{2}+\frac{c}{\varepsilon} h^{2}
$$

for any $\varepsilon>0$ by Assumptions (3.7), (3.8), and (1+). Here $c$ depends on the quantities $L^{*}$, $\left\|g_{t}\right\|_{C^{1,0}\left(\overline{\mathbb{S}}_{T}, \mathbb{R}^{2}\right)}:=\sup _{t \in(0, T)}\left\|g_{t}(\cdot, t)\right\|_{C^{1}\left(\mathbb{S}, \mathbb{R}^{2}\right)}, c_{\text {aprx }}, a_{*}^{-1}$, and $\beta^{*}:=\sup _{n \in \mathbb{S}} \beta(n)$ increasingly. With the help of

$$
\begin{gathered}
\left|\frac{\beta(N)}{a(N)}-\frac{\beta(n)}{a(n)}\right| \leqslant c\left(a_{*}^{-1}, \beta^{*},\|\beta, a\|_{W_{\infty}^{1}(\mathbb{S})}\right)|N-n|, \\
|N-n| \leqslant \frac{2}{\left|g_{u}\right|}\left|G_{u}-g_{u}\right|,\left.\quad|| g_{u}\right|^{2}-\left|G_{u}\right|^{2}\left|\leqslant c\left(L^{*}, l^{*}\right)\right| G_{u}-g_{u} \mid,
\end{gathered}
$$

we obtain

$$
I_{2} \leqslant \varepsilon\left\|G_{t}-J g_{t}\right\|_{L_{2}\left(\mathbb{S}, \mathbb{R}^{2}\right)}^{2}+\frac{c}{\varepsilon}\left\|G_{u}-g_{u}\right\|_{L_{2}\left(\mathbb{S}, \mathbb{R}^{2}\right)}^{2},
$$

where $c$ depends on the same quantities as in the estimate for $I_{3}$ and on $l_{*}^{-1}, l^{*},\|\beta, a\|_{W_{\infty}^{1}(\mathbb{S})}$ increasingly. The inequality

$$
\left|\frac{\left|G_{u}\right|}{a(N)} G_{u}^{\perp}-\frac{\left|g_{u}\right|}{a(n)} g_{u}^{\perp}\right| \leqslant c\left(L^{*}, l^{*}, a_{*}^{-1},\|a\|_{W_{\infty}^{1}(\mathbb{S})}\right)\left|G_{u}-g_{u}\right|
$$


yields

$$
I_{1} \leqslant \varepsilon\left\|G_{t}-J g_{t}\right\|_{L_{2}\left(\mathbb{S}, \mathbb{R}^{2}\right)}^{2}+\frac{c}{\varepsilon}\left(\left\|G_{u}-g_{u}\right\|_{L_{2}\left(\mathbb{S}, \mathbb{R}^{2}\right)}^{2}+\|\Theta(G)-\theta(g)\|_{L_{2}(\Omega)}^{2}\right),
$$

where $c$ depends on $\|\theta\|_{C^{0}(\bar{Q})}$ additionally. Besides,

$$
\left\|G_{t}-g_{t}\right\|_{L_{2}\left(\mathbb{S}, \mathbb{R}^{2}\right)}^{2} \leqslant 2\left\|G_{t}-J g_{t}\right\|_{L_{2}\left(\mathbb{S}, \mathbb{R}^{2}\right)}^{2}+c h^{2} .
$$

We collect the above estimates, choose $\varepsilon>0$ sufficiently small and get

$$
\begin{aligned}
\frac{\beta_{*} L_{*}}{a^{*}} \int_{0}^{t}\left\|G_{t}-g_{t}\right\|_{L_{2}\left(\mathbb{S}, \mathbb{R}^{2}\right)}^{2} & +\left\|G_{u}(t)-g_{u}(t)\right\|_{L_{2}\left(\mathbb{S}, \mathbb{R}^{2}\right)}^{2} \\
& \leqslant c\left[\int_{0}^{t}\left\|G_{u}-g_{u}\right\|_{L_{2}\left(\mathbb{S}, \mathbb{R}^{2}\right)}^{2}+\int_{0}^{t}\|\Theta(G)-\theta(g)\|_{L_{2}(\mathbb{S})}^{2}+h^{2}\right]
\end{aligned}
$$

for any $t \in\left[0, t^{*}\right]$ by integrating in time and the approximation of $g_{0}$. Here $c$ depends on $L_{*}^{-1}, \beta_{*}^{-1}$, $a^{*}, \int_{0}^{T}\left\|g_{t}\right\|_{H^{2}\left(\mathbb{S}, \mathbb{R}^{2}\right)}^{2}$ and $\left\|g_{0}\right\|_{C^{2}\left(\mathbb{S}, \mathbb{R}^{2}\right)}$ additionally. The last inequality and

$$
\|G(t)-g(t)\|_{L_{2}\left(\mathbb{S}, \mathbb{R}^{2}\right)}^{2} \leqslant e^{T}\left(c h^{2}+\int_{0}^{t}\left\|G_{t}-g_{t}\right\|_{L_{2}\left(\mathbb{S}, \mathbb{R}^{2}\right)}^{2}\right)
$$

for all $t \in[0, T]$, a consequence of the Gronwall Lemma and the approximation of $g_{0}$, conclude the proof.

\section{Control of the temperature field (Part 1)}

A main issue in estimating the error of the temperature field is an appropriate control of $\varphi(g)-$ $\Phi(G)$, where $\Phi \in X$ is the test function in the equation for $\Theta$ in the semi-discrete Problem 2.2 and $\varphi \in H^{1}(\Omega)$ is the test function in the corresponding equation for $\theta$. In contrary to the usual choice $\varphi=\Phi$, we propose to proceed as follows: construct a mapping $H: \bar{\Omega} \times\left[0, t^{*}\right] \rightarrow \bar{\Omega}$ such that

$$
H(\cdot, t) \text { is bijective and } H(g(u, t), t)=G(u, t)
$$

for all $u \in \mathbb{S}$ and $t \in\left[0, t^{*}\right]$. Let us denote the inverse of $H(\cdot, t)$ by $H^{-1}(\cdot, t)$. Choosing $\varphi=$ $\Phi \circ H(\cdot, t)$ as the continuous test function, we calculate formally that $\hat{\theta}:=\theta \circ H^{-1}$, more precisely $\hat{\theta}(x, t)=\theta\left(H^{-1}(x, t), t\right)$, fulfills for almost every $t \in\left[0, t^{*}\right]$

$$
\int_{\Omega} \hat{\theta}_{t} \Phi+\int_{\Omega} \nabla \hat{\theta} \cdot \nabla \Phi=-\int_{\mathbb{S}} g_{t} \cdot g_{u}^{\perp} \Phi(G)+\mathcal{E}(\Phi),
$$

where

$$
\begin{aligned}
\mathcal{E}(\Phi):= & \int_{\Omega}(\nabla \theta) \circ H^{-1}\left(H^{-1}\right)_{t} \Phi+\int_{\Omega} \theta_{t} \Phi \circ H(\mathbb{J} H-1) \\
& +\int_{\Omega}(\nabla \theta) \circ H^{-1}\left(D\left(H^{-1}\right)-E\right) \cdot \nabla \Phi+\int_{\Omega} \nabla \theta \cdot(\nabla \Phi) \circ H(\mathbb{J} H-1) \\
& \quad+\int_{\Omega} \nabla \theta \cdot(\nabla \Phi) \circ H(E-D H) .
\end{aligned}
$$

Hereafter $\mathbb{J} H:=|\operatorname{det} D H|$ and $E$ denotes the matrix of the identity in $\mathbb{R}^{2}$. In doing so we need not to control $\varphi(g)-\Phi(G)$, but we have to estimate $\mathcal{E}$ and then $\Theta-\hat{\theta}$ and $\hat{\theta}-\theta$. 


\section{Construction and control of the mapping $\boldsymbol{H}$}

In order to carry out the above proposal we have to give a concrete construction of $H$. A useful tool for this is the following lemma, the qualitative version of which is well known. Define

$$
\bar{\kappa}(f):=\sup _{u \in \mathbb{S}}|k(u)| \quad \text { and } \quad \underline{Q}(f):=\inf \left\{\frac{\left|x_{1}-x_{2}\right|}{l\left(x_{1}, x_{2}\right)} \mid x_{1}, x_{2} \in f(\mathbb{S}), x_{1} \neq x_{2}\right\},
$$

where $f: \mathbb{S} \rightarrow \mathbb{R}^{2}$ is a regular $C^{2}$-parametrization, $k(u)$ is the curvature of $f(\mathbb{S})$ in $f(u)$, and $l\left(x_{1}, x_{2}\right)$ denotes the length of the shortest path in $f(\mathbb{S})$ that connects $x_{1}$ and $x_{2}$. Note, that we have

$$
\frac{\mathrm{DQ}(f)}{\sup _{\mathbb{S}}\left|f_{u}\right|} \leqslant \underline{Q}(f) \leqslant \frac{\underline{\mathrm{DQ}}(f)}{\inf _{\mathbb{S}}\left|f_{u}\right|}
$$

and equality, if the length element $\left|f_{u}\right|$ is constant. Besides, we define $U_{R}(A):=\left\{x \in \mathbb{R}^{2} \mid\right.$ $\operatorname{dist}(A, x)<R$ f for any $R>0$ and $A \subset \mathbb{R}^{2}$.

LEMMA 4.2 Let $f$ be a regular $C^{2}$-parametrization and set $R:=\underline{Q}(f) / \bar{\kappa}(f)>0$. Then $\mathcal{F}$ : $(-R, R) \times \mathbb{S} \rightarrow U_{R}(f(\mathbb{S}))$, defined by

$$
\mathcal{F}(d, u):=f(u)-d \frac{f_{u}(u)^{\perp}}{\left|f_{u}(u)\right|},
$$

is a $C^{1}$-diffeomorphism with sdist $(f, \mathcal{F}(d, u))=d$ for all $(d, u) \in(-R, R) \times \mathbb{S}$.

Proof. See [29, Satz 10.13].

Consider the mapping

$$
\mathcal{G}(d, u, t):=g(u, t)+d n(u, t), \quad d \in \mathbb{R}, u \in \mathbb{S}, t \in[0, T],
$$

and set

$$
\begin{gathered}
R_{*}:=\inf _{t \in(0, T)} \underline{Q}(g(t)) / \bar{\kappa}(g(t)), \quad \operatorname{dist}_{*}:=\inf _{t \in(0, T)} \operatorname{dist}\left(g(\mathbb{S}, t), \mathbb{R}^{2} \backslash \Omega\right), \\
d^{*}:=\min \left\{1, R_{*}, \operatorname{dist}_{*}\right\} .
\end{gathered}
$$

Assumption (3.9) guarantees that $d^{*}>0$. For any $t \in(0, T)$ we have that $U_{d^{*}}(g(\mathbb{S}, t)) \subset \Omega$ and that the restriction of $\mathcal{G}(\cdot, t)$ on $M_{d^{*}}:=\left(-d^{*}, d^{*}\right) \times \mathbb{S}$ is a $C^{1}$-diffeomorphism onto $U_{d^{*}}(g(\mathbb{S}, t))$ by Lemma 4.2. Moreover,

$$
\begin{gathered}
\frac{1}{2} l_{*} \leqslant J \mathcal{G}(d, u, t)=(1+d k(u, t))\left|g_{u}(u, t)\right| \leqslant \frac{3}{2} l^{*}, \\
|D \mathcal{G}(d, u, t)| \leqslant \max \left\{1, \frac{3}{2} l^{*}\right\}
\end{gathered}
$$

for any $(d, u) \in M_{d^{*} / 2}, t \in[0, T]$ and

$$
\left|D\left(\mathcal{G}^{-1}\right)(x, t)\right| \leqslant\left(\min \left\{1, \frac{1}{2} l_{*}\right\}\right)^{-1}
$$


for any $x \in U_{d^{*} / 2}(g(\mathbb{S}, t)), t \in[0, T]$. A further investigation shows that

$$
\begin{gathered}
\mathcal{G} \in C^{1}\left(M_{d^{*}} \times(0, T), \mathbb{R}^{2}\right), \quad \mathcal{G}^{-1} \in C^{1}\left(\mathcal{U}_{d^{*}}, \mathbb{R} \times \mathbb{S}\right), \\
D \mathcal{G} \in L_{2}\left(0, T ; H^{1}\left(M_{d^{*}}, \mathbb{R}^{4}\right)\right), \quad \mathcal{G}_{t} \in L_{2}\left(0, T ; H^{1}\left(M_{d^{*}}, \mathbb{R}^{2}\right)\right)
\end{gathered}
$$

with $\mathcal{U}_{d^{*}}:=\bigcup_{t \in(0, T)} U_{d^{*}}(g(\mathbb{S}, t)) \times\{t\}$ open and

$$
\left|\mathcal{G}_{t}(d, u, t)\right| \leqslant\left\|g_{t}\right\|_{C^{0}\left(\overline{\mathbb{S}}_{T}, \mathbb{R}^{2}\right)}+\frac{|d|}{l_{*}}\left\|g_{u t}\right\|_{C^{0}\left(\overline{\mathbb{S}}_{T}, \mathbb{R}^{2}\right)}
$$

for every $(d, u) \in \mathbb{R} \times \mathbb{S}, t \in[0, T]$,

$$
\begin{gathered}
\left|\partial_{d} D \mathcal{G}(d, u, t)\right| \leqslant \kappa^{*} l^{*}, \\
\left|\partial_{u} D \mathcal{G}(d, u, t)\right| \leqslant c\left(l_{*}^{-1},\left\|g_{t}\right\|_{C^{1,0}\left(\overline{\mathbb{S}}_{T}, \mathbb{R}^{2}\right)}\right)\left(1+d\left(1+\left|g_{\text {uиu }}(u, t)\right|\right)\right), \\
\left|D \mathcal{G}_{t}(d, u, t)\right| \leqslant c\left(l_{*}^{-1},\left\|g_{u}\right\|_{C^{1}\left(\overline{\mathbb{S}}_{T}, \mathbb{R}^{2}\right)}\right)\left(1+d^{2}\left(1+\left|g_{\text {tuu }}(u, t)\right|^{2}\right)\right)^{1 / 2}
\end{gathered}
$$

for every $d \in \mathbb{R}$, almost every $(u, t) \in \mathbb{S}_{T}$ with $\kappa^{*}:=\sup _{t \in(0, T)} \bar{\kappa}(g(t))$.

Let $\eta \in C^{1}(\mathbb{R})$ be a cut-off function with $\eta(d)=1$ for all $|d| \leqslant \frac{1}{4} d^{*}, \eta(d)=0$ for all $|d| \geqslant \frac{1}{2} d^{*}, 0 \leqslant \eta \leqslant 1$ and $\left|\eta^{\prime}\right| \leqslant 8 / d^{*}$ on $\mathbb{R}$.

DEFINITION 4.1 Suppose that

$$
\|G-g\|_{C^{0}\left(\mathbb{S}, \mathbb{R}^{2}\right)} \leqslant \frac{1}{4} d^{*} \quad \text { in }\left[0, t^{*}\right] .
$$

Define the auxiliary mappings $\rho: \mathbb{S} \times\left[0, t^{*}\right] \rightarrow \mathbb{R}$ and $\sigma: \mathbb{S} \times\left[0, t^{*}\right] \rightarrow \mathbb{S}$ by $G=\mathcal{G}(\rho, \sigma, t)$ or, more precisely, by the equation

$$
G(u, t)=\mathcal{G}(\rho(u, t), \sigma(u, t), t) .
$$

In addition, define a further auxiliary mapping $\mathcal{H}: \mathbb{R} \times \mathbb{S} \times\left[0, t^{*}\right] \rightarrow \mathbb{R} \times \mathbb{S}$ by

$$
\mathcal{H}(d, u, t):=\left(d+\eta(d) \rho(u, t), u e^{i \phi}\right) \quad \text { with } \quad \phi=\eta(d) \arg \left(\frac{\sigma(u, t)}{u}\right),
$$

where we use $\mathbb{R}^{2} \simeq \mathbb{C}$ and arg : $\mathbb{C} \backslash\{0\} \rightarrow(-\pi, \pi]$ denotes the argument function. Finally, define $H: \Omega \times\left[0, t^{*}\right] \rightarrow \Omega$ by

$$
H(x, t):= \begin{cases}\mathcal{G}\left(\mathcal{H}\left(\mathcal{G}^{-1}(x, t), t\right), t\right), & \text { if } x \in U_{d^{*}}(g(\mathbb{S}, t)) \\ x, & \text { otherwise. }\end{cases}
$$

Assumption (2+) guarantees that $\rho, \sigma$ and thus $H$ are well defined. The construction in Definition 4.1 satisfies $H(g)=G$. The rest of this subsection is devoted to the following issues: the construction of $H$ can be controlled by the error of the free boundary, and (therefore) $H$ is bijective and the formal calculations that have led to (4.1) can be done rigorously provided that the error of the free boundary is sufficiently small. 
The next lemma concerns the first aforementioned issue. More precisely, it establishes that the 'distance' between the auxiliary mapping $\mathcal{H}(\cdot, t), t \in\left[0, t^{*}\right]$, and the identity on $\mathbb{R} \times \mathbb{S}$ is controlled by the error of the free boundary in a 'pointwise' sense. To this end, define a metric on $\mathbb{R} \times \mathbb{S}$ by

$$
d_{\mathbb{R} \times \mathbb{S}}\left(\left(d_{1}, u_{1}\right),\left(d_{2}, u_{2}\right)\right):=\left(\left|d_{1}-d_{2}\right|^{2}+d_{\mathbb{S}}\left(u_{1}, u_{2}\right)^{2}\right)^{1 / 2}
$$

and set

$$
\mathrm{DQ}_{*}:=\inf _{t \in(0, T)} \underline{\mathrm{DQ}}(g(t))>0 .
$$

Lemma 4.3 Suppose that (2+) in Definition 4.1 and

$$
\|G-g\|_{C^{0}\left(\mathbb{S}, \mathbb{R}^{2}\right)} \leqslant \frac{\mathrm{DQ}_{*}}{4} \pi \quad \text { in }\left[0, t^{*}\right]
$$

hold. Then:

(i) We have $\mathcal{H} \in C^{0}\left(\mathbb{R} \times \mathbb{S} \times\left[0, t^{*}\right], \mathbb{R} \times \mathbb{S}\right)$ with

$$
d_{\mathbb{R} \times \mathbb{S}}(\mathcal{H}(d, u, t),(d, u)) \leqslant\left(1+\frac{4}{\mathrm{DQ}_{*}^{2}}\right)^{1 / 2}|G(u, t)-g(u, t)| .
$$

(ii) Let $t \in\left[0, t^{*}\right]$. We have $\mathcal{H}(\cdot, t) \in W_{\infty}^{1}\left(M_{d}, \mathbb{R} \times \mathbb{S}\right)$ for any $d>0$ and

$$
|D \mathcal{H}(d, u, t)-E| \leqslant c\left(\left|G_{u}(u, t)-g_{u}(u, t)\right|^{2}+|G(u, t)-g(u, t)|^{2}\right)^{1 / 2}
$$

for every $d \in \mathbb{R}$ and almost every $u \in \mathbb{S}$. Here $c$ depends on $\mathrm{DQ}_{*}^{-1}$, dist $_{*}^{-1}$, and $\left\|g_{u}\right\|_{C^{1,0}\left(\overline{\mathbb{S}}_{T}, \mathbb{R}^{2}\right)}$ increasingly.

(iii) Let $u \in \mathbb{S}$. The mapping $\mathbb{R} \times\left[0, t^{*}\right] \ni(d, t) \mapsto \mathcal{H}(d, u, t) \in \mathbb{R} \times \mathbb{S}$ is continuously differentiable and we have

$$
\left|\mathcal{H}_{t}(d, u, t)\right| \leqslant c\left(\left|G_{t}(u, t)-g_{t}(u, t)\right|^{2}+|G(u, t)-g(u, t)|^{2}\right)^{1 / 2}
$$

for every $d \in \mathbb{R}$ and $t \in\left[0, t^{*}\right]$. Here $c$ depends on $\mathrm{DQ}_{*}^{-1}$ and $\left\|g_{t u}\right\|_{C^{0}\left(\overline{\mathbb{S}}_{T}, \mathbb{R}^{2}\right)}$ increasingly.

Proof. Let us prove statement (i). We first derive corresponding statements on the auxiliary mappings $\rho$ and $\sigma$. With the help of $G \in C^{0}\left(\mathbb{S} \times\left[0, t^{*}\right], \mathbb{R}^{2}\right)$, Assumption (2+) and (4.2d) we obtain $\rho \in C^{0}\left(\mathbb{S} \times\left[0, t^{*}\right]\right)$ as well as $\sigma \in C^{0}\left(\mathbb{S} \times\left[0, t^{*}\right], \mathbb{S}\right)$. Besides, for any $u \in \mathbb{S}$ and $t \in\left[0, t^{*}\right]$ we calculate

$$
|\rho(u, t)|=|\operatorname{sdist}(g, G(u, t))|=\operatorname{dist}(g(\mathbb{S}, t), G(u, t)) \leqslant|G(u, t)-g(u, t)|
$$

by using Lemma 4.2. Hence,

$$
\begin{aligned}
d_{\mathbb{S}}(\sigma(u, t), u) \leqslant \underline{\mathrm{DQ}}(g(t))^{-1} \mid G(u, t)-\rho(u, t) n(\sigma(u, t), t) & -g(u, t) \mid \\
& \leqslant \frac{2}{\mathrm{DQ}_{*}}|G(u, t)-g(u, t)| .
\end{aligned}
$$


We thus have $|\arg (\sigma(u, t) / u)|=d_{\mathbb{S}}(\sigma(u, t), u)<\pi$ in $\mathbb{S} \times\left[0, t^{*}\right]$ by Assumption (3+) and the function

$$
\mathbb{S} \times\left[0, t^{*}\right] \ni(u, t) \mapsto \arg (\sigma(u, t) / u) \in \mathbb{R} \quad \text { is continuous. }
$$

Together with the continuity of $\rho, \sigma$, and $\eta$, this implies $\mathcal{H} \in C^{0}\left(\mathbb{R} \times \mathbb{S} \times\left[0, t^{*}\right], \mathbb{R} \times \mathbb{S}\right)$. The inequality in statement (i) follows from (4.3), (4.4), and $0 \leqslant \eta \leqslant 1$. Statement (i) thus is established.

Let us prove statement (ii). As before, we first derive corresponding statements on $\rho$ and $\sigma$. Let $t \in\left[0, t^{*}\right]$. The facts $\mathcal{G}^{-1}(\cdot, t) \in C^{1}\left(\overline{U_{d^{*} / 2}(g(\mathbb{S}, t))}\right)$ and $G(\cdot, t) \in W_{\infty}^{1}\left(\mathbb{S}, \mathbb{R}^{2}\right)$ imply $\rho(\cdot, t) \in W_{\infty}^{1}(\mathbb{S})$ and $\sigma(\cdot, t) \in W_{\infty}^{1}(\mathbb{S}, \mathbb{S})$. Moreover, the chain rule yields (recall the convention for derivatives)

$$
\begin{aligned}
G_{u}(u, t)-g_{u}(u, t)=n & (\sigma(u, t), t) \rho_{u}(u, t) \\
& +(1+\rho(u, t) k(\sigma(u, t), t)) g_{u}(\sigma(u, t), t) \sigma_{u}(u, t)-g_{u}(u, t) .
\end{aligned}
$$

In order to estimate $\left|\rho_{u}(u, t)\right|$ we take the scalar product of $(4.6)$ and $n(\sigma(u, t), t)$ and arrive at

$$
\left|\rho_{u}(u, t)\right| \leqslant\left|G_{u}(u, t)-g_{u}(u, t)\right|+\left|g_{u}(\sigma(u, t))-g_{u}(u, t)\right| .
$$

With the help of (4.4) we estimate

$$
\left|g_{u}(\sigma(u, t))-g_{u}(u, t)\right| \leqslant \frac{2\left\|g_{u u}\right\|_{C^{0}\left(\overline{\mathbb{S}}_{T}, \mathbb{R}^{2}\right)}}{\mathrm{DQ}_{*}}|G(u, t)-g(u, t)| .
$$

Inserting this inequality in the previous one, we get

$$
\left|\rho_{u}(u, t)\right| \leqslant c\left(\left|G_{u}(u, t)-g_{u}(u, t)\right|+|G(u, t)-g(u, t)|\right),
$$

where $c$ depends on $\mathrm{DQ}_{*}^{-1}$ and $\left\|g_{u u}\right\|_{C^{0}\left(\overline{\mathbb{S}}_{T}, \mathbb{R}^{2}\right)}$ increasingly. Next, let us estimate the expression $\left|\sigma_{u}(u, t)-1\right|$. We take the scalar product of (4.6) and $n(\sigma(u, t), t)^{\perp}$, use (4.2a) and $|k(u, t)| \leqslant$ $\left|g_{u u}(u, t)\right| /\left|g_{u}(u, t)\right|^{2}$ in order to get

$$
\frac{1}{2} l_{*}\left|\sigma_{u}(u, t)-1\right| \leqslant\left|G_{u}(u, t)-g_{u}(u, t)\right|+\frac{\left|g_{u u}(u, t)\right|}{\left|g_{u}(u, t)\right|}|\rho(u, t)|+\left|g_{u}(\sigma(u, t))-g_{u}(u, t)\right| .
$$

This implies

$$
\left|\sigma_{u}(u, t)-1\right| \leqslant c\left(\left|G_{u}(u, t)-g_{u}(u, t)\right|+|G(u, t)-g(u, t)|\right)
$$

with $c=c\left(\mathrm{DQ}_{*}^{-1},\left\|g_{u u}\right\|_{C^{0}\left(\overline{\mathbb{S}}_{T}, \mathbb{R}^{2}\right)}\right)$ because of $\left|g_{u}(u, t)\right| \geqslant \mathrm{DQ}_{*}$, (4.3) and (4.7). The regularity of $\rho, \sigma$, and $\eta$ yield $\mathcal{H}(\cdot, t) \in W_{\infty}^{1}\left(M_{d}, \mathbb{R} \times \mathbb{S}\right)$ for any $d>0$. Moreover, choosing the basis $(1,0),\left(0, u^{\perp}\right)$ in $T(\mathbb{R} \times \mathbb{S})_{(d, u)}$, we have

$$
D \mathcal{H}(d, u, t)=\left(\begin{array}{cc}
1+\eta^{\prime}(d) \rho(u, t) & \eta(d) \rho_{u}(u, t) \\
\eta^{\prime}(d) \arg (\sigma(u, t) / u) & 1+\eta(d)\left(\sigma_{u}(u, t)-1\right)
\end{array}\right)
$$


for all $d \in \mathbb{R}$ and a.e. $u \in \mathbb{S}$. Therefore, inequalities (4.3), (4.4), (4.8), (4.9), and the properties of $\eta$ imply

$$
|D \mathcal{H}(d, u, t)-E| \leqslant c\left(\left|G_{u}(u, t)-g_{u}(u, t)\right|^{2}+|G(u, t)-g(u, t)|^{2}\right)^{1 / 2}
$$

where $c$ depends on $\left(d^{*}\right)^{-1}$ additionally. The proof of statement (ii) is complete; statement (iii) is established in a similar manner.

The next lemma is the key step in establishing that $H$ is bijective.

Lemma 4.4 Let $c_{0}$ be the constant in statement (ii) of Lemma 4.3. Suppose in addition to (2+) and (3+) that

$$
\|G-g\|_{W_{\infty}^{1}\left(\mathbb{S}, \mathbb{R}^{2}\right)} \leqslant \frac{1}{\sqrt{8} c_{0}} \quad \text { in }\left[0, t^{*}\right]
$$

Then the mapping $\mathcal{H}(\cdot, t)$ is bilipschitz (that is $\mathcal{H}(\cdot, t)$ and $\mathcal{H}^{-1}(\cdot, t):=(\mathcal{H}(\cdot, t))^{-1}$ are Lipschitz continuous) for any $t \in\left[0, t^{*}\right]$ and we have

$$
\begin{aligned}
|D \mathcal{H}(\cdot, t)| \leqslant \frac{3}{2}, & \left|D\left(\mathcal{H}^{-1}\right)(\cdot, t)\right| \leqslant 2, \quad \frac{1}{4} \leqslant \mathbb{J} \mathcal{H}(\cdot, t)=\operatorname{det} D \mathcal{H}(\cdot, t) \leqslant \frac{9}{4}, \\
& D\left(\mathcal{H}^{-1}\right)(\cdot, t)=\left[D \mathcal{H}\left(\mathcal{H}^{-1}(\cdot, t), t\right)\right]^{-1}
\end{aligned}
$$

almost everywhere in $\mathbb{R} \times \mathbb{S}$. Moreover, for every $(d, u) \in \mathbb{R} \times \mathbb{S}$ the mappings $\mathcal{H}(d, u, \cdot)$ and $\mathcal{H}^{-1}(d, u, \cdot)$ are Lipschitz and their Lipschitz constants do not depend on $(d, u)$.

Proof. Define a group operation on $\mathbb{R} \times \mathbb{S}$ by $\left(d_{1}, u_{1}\right)+\left(d_{2}, u_{2}\right):=\left(d_{1}+d_{2}, u_{1} u_{2}\right)$ and denote the identity on $\mathbb{R} \times \mathbb{S}$ with $\mathcal{I}$. Let $t \in\left[0, t^{*}\right]$. Assumption (4+) implies that $\mathcal{H}(\cdot, t)-\mathcal{I}$ is a contraction on $\mathbb{R} \times \mathbb{S}$ with Lipschitz constant $\frac{1}{2}$. From this fact and the observation that the distance and group operation on $\mathbb{R} \times \mathbb{S}$ are 'compatible' one derives that $\mathcal{H}(\cdot, t)$ is bijective, that $\mathcal{H}^{-1}(\cdot, t):=(\mathcal{H}(\cdot, t))^{-1}$ is Lipschitz and that the inequalities for $|D \mathcal{H}(\cdot, t)|,\left|D\left(\mathcal{H}^{-1}\right)(\cdot, t)\right|$ and $\mathbb{J} \mathcal{H}(\cdot, t)$ are satisfied. The formula for $D\left(\mathcal{H}^{-1}\right)(\cdot, t)$ is a consequence of [14,3.1.2, Corollary 1 (ii)]. Choose $(d, u) \in \mathbb{R} \times \mathbb{S}$ such that this formula is valid. Then $\operatorname{det} D \mathcal{H}(d, u, t)>0$, because $[0,1] \ni \alpha \mapsto \alpha D \mathcal{H}(d, u, t)+(1-\alpha) E$ is a continuous path in the regular matrices. Thus, we have $\mathbb{J H}(\cdot, t)=\operatorname{det} D \mathcal{H}(\cdot, t)$ almost everywhere.

Let $(d, u) \in \mathbb{R} \times \mathbb{S}$ be arbitrary. Lemma 4.3 (iii) and $G \in C^{1}\left(\left[0, t^{*}\right], Y\right)$ imply that $\mathcal{H}(d, u, \cdot)$ is Lipschitz, and its Lipschitz constant does not depend on $(d, u)$. Following the lines of [2, (3.1.4)] one proves that the last statement implies the corresponding one on $\mathcal{H}^{-1}(d, u, \cdot)$.

The statements on $\mathcal{H}$ in Lemma 4.4 imply analogous ones on $H$ provided the assumptions $(2+)-(4+)$ hold; this can be proved by using the statements (4.2) on the diffeomorphisms $\mathcal{G}(\cdot, t)$, $t \in[0, T]$. Moreover, one can verify the following formulae:

$$
\begin{gathered}
D H(x, t)=D \mathcal{G}\left(\mathcal{H}\left(\mathcal{G}^{-1}(x, t), t\right), t\right) D \mathcal{H}\left(\mathcal{G}^{-1}(x, t), t\right) D\left(\mathcal{G}^{-1}\right)(x, t), \\
D\left(H^{-1}\right)(x, t)=\left[D H\left(H^{-1}(x, t), t\right)\right]^{-1}
\end{gathered}
$$


for almost every $x \in U_{d^{*} / 2}(g(\mathbb{S}, t))$ and every $t \in\left[0, t^{*}\right]$, and

$$
\begin{aligned}
H_{t}=D \mathcal{G} \circ \mathcal{H} \circ \mathcal{G}^{-1} D \mathcal{H} \circ \mathcal{G}^{-1}\left(\mathcal{G}^{-1}\right)_{t} \\
\quad+D \mathcal{G} \circ \mathcal{H} \circ \mathcal{G}^{-1} \mathcal{H}_{t} \circ \mathcal{G}^{-1}+\mathcal{G}_{t} \circ \mathcal{H} \circ \mathcal{G}^{-1}, \\
\left(H^{-1}\right)_{t}=-\left([D H]^{-1} H_{t}\right) \circ H^{-1}
\end{aligned}
$$

almost everywhere in $\mathcal{U}_{d^{*} / 2}$. In addition, the chain rule [32, Theorem 2.2.2] and the trace theorem [14, 4.3, Theorem 1 (with Remark)] lead to

$$
\begin{aligned}
& \nabla(\varphi \circ H(t))=(\nabla \varphi) \circ H(t) D H(t), \\
& \varphi \in \stackrel{\circ}{H}^{1}(\Omega) \Longrightarrow \varphi \circ H(t) \in \stackrel{\circ}{H}^{1}(\Omega)
\end{aligned}
$$

for every $\varphi \in H^{1}(\Omega), t \in\left[0, t^{*}\right]$, and $\theta \circ H^{-1} \in W_{\infty}^{1}\left(\Omega \times\left[0, t^{*}\right]\right)$ with

$$
\left(\theta \circ H^{-1}\right)_{t}=(\nabla \theta) \circ H^{-1}\left(H^{-1}\right)_{t}+\theta_{t} \circ H^{-1} \text {. }
$$

Therefore, (4.1) is valid for every $\Phi \in X^{\circ}$ and almost every $t \in\left[0, t^{*}\right]$.

\section{Control of the temperature field (Part 2)}

In this subsection we aim at estimating $\Theta-\hat{\theta}$ in terms of the error of the free boundary. In order to reach such an estimate we split the difference $\Theta-\hat{\theta}$ in the following way. Let $\Lambda \hat{\theta}$ be the parabolic projection (compare with [21]) of $\hat{\theta}$ onto $H^{1}\left(0, t^{*} ; \dot{X}\right)$, that is $\Lambda \hat{\theta} \in H^{1}\left(0, t^{*} ; \dot{X}\right)$ solves

$$
\begin{gathered}
\int_{\Omega}(\Lambda \hat{\theta})_{t} \Phi+\int_{\Omega} \nabla(\Lambda \hat{\theta}) \cdot \nabla \Phi=\int_{\Omega} \hat{\theta}_{t} \Phi+\int_{\Omega} \nabla \hat{\theta} \cdot \nabla \Phi \quad \forall \Phi \in \dot{X}, \text { a.e. in }\left(0, t^{*}\right), \\
(\Lambda \hat{\theta})(0)=\hat{\theta}(0)=0,
\end{gathered}
$$

and write

$$
\Theta-\hat{\theta}=(\Theta-\Lambda \hat{\theta})+(\Lambda \hat{\theta}-\hat{\theta}) .
$$

Let us estimate $\Theta-\Lambda \hat{\theta}$ first. Equation (4.1) remains valid, if we substitute $\hat{\theta}$ by $\Lambda \hat{\theta}$. We test the equation for $\Theta$ in Problem 2.2 and (4.1) for $\Lambda \hat{\theta}$ with $\Phi=\Theta-\Lambda \hat{\theta}$, take their difference, integrate in time, and get for any $t \in\left[0, t^{*}\right]$

$$
\frac{1}{2}\|\Theta(t)-\Lambda \hat{\theta}(t)\|_{L_{2}(\Omega)}^{2}+\int_{0}^{t}\|\nabla(\Theta-\Lambda \hat{\theta})\|_{L_{2}(\Omega)}^{2} \leqslant \mathcal{I}+\int_{0}^{t} \mathcal{E}(\Theta-\Lambda \hat{\theta})
$$

where

$$
\mathcal{I}:=-\int_{0}^{t} \int_{\mathbb{S}}\left(G_{t} \cdot G_{u}^{\perp}-g_{t} \cdot g_{u}^{\perp}\right)(\Theta-\Lambda \hat{\theta})(G) .
$$

We proceed with two lemmata. The first one concerns $\mathcal{E}$ and the second one $\mathcal{I}$. 
LEMMA 4.5 Suppose that $(2+)-(4+)$ hold. Then,

$$
\begin{aligned}
|\mathcal{E}(\varphi)| \leqslant c\left(\mathcal{A}\|G-g\|_{H^{1}\left(\mathbb{S}, \mathbb{R}^{2}\right)}+\left\|G_{t}-g_{t}\right\|_{L_{2}\left(\mathbb{S}, \mathbb{R}^{2}\right)}\right) & \|\varphi\|_{L_{2}(\Omega)} \\
& +c \mathcal{A}\|G-g\|_{H^{1}\left(\mathbb{S}, \mathbb{R}^{2}\right)}\|\nabla \varphi\|_{L_{2}(\Omega)}
\end{aligned}
$$

for every $\varphi \in \stackrel{H}{ }^{1}(\Omega)$ and almost every $t \in\left[0, t^{*}\right]$. Here the constant $c$ depends on $\mathrm{DQ}_{*}^{-1}$, dist ${ }_{*}^{-1}$, $\left\|g_{u}\right\|_{C^{1}\left(\overline{\mathbb{S}}_{T}, \mathbb{R}^{2}\right)},\left\|g_{t}\right\|_{C^{0}\left(\overline{\mathbb{S}}_{T}, \mathbb{R}^{2}\right)},\left\|\nabla \theta, \theta_{t}\right\|_{L_{\infty}(Q)}$ increasingly and

$$
\mathcal{A}:=\left(1+\left\|g_{\text {tuu }}\right\|_{L_{2}\left(\mathbb{S}, \mathbb{R}^{2}\right)}^{2}+\left\|g_{\text {uиu }}\right\|_{L_{2}\left(\mathbb{S}, \mathbb{R}^{2}\right)}^{2}\right)^{1 / 2} .
$$

Proof. We only estimate the first integral in the definition of $\mathcal{E}$; the estimates of the other integrals are more straight-forward. Let $t \in\left[0, t^{*}\right]$ and drop it normally. Since

$$
\left|\int_{\Omega}(\nabla \theta) \circ H^{-1}\left(H^{-1}\right)_{t} \varphi\right| \leqslant\|\nabla \theta\|_{L_{\infty}(\Omega)}\left\|\left(H^{-1}\right)_{t}\right\|_{L_{2}(\Omega)}\|\varphi\|_{L_{2}(\Omega)},
$$

we have to estimate $\left\|\left(H^{-1}\right)_{t}\right\|_{L_{2}(\Omega)}$. Using (4.10d) and $\left(H^{-1}\right)_{t}=0$ off $U_{d^{*} / 2}(g(\mathbb{S}, t))$, we get

$$
\left\|\left(H^{-1}\right)_{t}\right\|_{L_{2}(\Omega)} \leqslant c\left(l_{*}^{-1}, l^{*}\right) \int_{M_{d^{*} / 2}}\left|\left([D H]^{-1} H_{t}\right) \circ \mathcal{G}\right|^{2} .
$$

Formulae (4.10a) and (4.10c) yield $\left([D H]^{-1} H_{t}\right) \circ \mathcal{G}=\sum_{i=1}^{4} D_{i}$, where

$$
\begin{aligned}
D_{1} & :=D \mathcal{G}[D \mathcal{H}]^{-1} \mathcal{H}_{t}, \\
D_{2} & :=D \mathcal{G}[D \mathcal{H}]^{-1}(E-D \mathcal{H})\left([D \mathcal{G}]^{-1} \mathcal{G}_{t}\right) \circ \mathcal{H}, \\
D_{3} & :=D \mathcal{G}[D \mathcal{G}]^{-1} \circ \mathcal{H}(D \mathcal{G}-D \mathcal{G} \circ \mathcal{H})[D \mathcal{G}]^{-1} \mathcal{G}_{t} \circ \mathcal{H}, \\
D_{4} & :=\mathcal{G}_{t} \circ \mathcal{H}-\mathcal{G}_{t} .
\end{aligned}
$$

We estimate $D_{1}, D_{2}, D_{3}$, and $D_{4}$ in the $\|\cdot\|_{L_{2}\left(M_{d^{*} / 2}, \mathbb{R}^{2}\right)}$-norm by using the inequalities of the previous subsection. We establish

$$
\int_{M_{d^{*} / 2}}\left|D_{1}\right|^{2} \leqslant c\left(\left\|G_{t}-g_{t}\right\|_{L_{2}\left(\mathbb{S}, \mathbb{R}^{2}\right)}^{2}+\|G-g\|_{L_{2}\left(\mathbb{S}, \mathbb{R}^{2}\right)}^{2}\right)
$$

with $c=c\left(\mathrm{DQ}_{*}^{-1}, l^{*},\left\|g_{t u}\right\|_{C^{0}\left(\overline{\mathbb{S}}_{T}, \mathbb{R}^{2}\right)}\right)$ and

$$
\int_{M_{d^{*} / 2}}\left|D_{2}\right|^{2} \leqslant c\left(\mathrm{DQ}_{*}^{-1}, \operatorname{dist}_{*}^{-1},\left\|g_{t}\right\|_{C^{0}\left(\overline{\mathbb{S}}_{T}, \mathbb{R}^{2}\right)},\left\|g_{u}\right\|_{C^{1}\left(\overline{\mathbb{S}}_{T}, \mathbb{R}^{2}\right)}\right)\|G-g\|_{H^{1}\left(\mathbb{S}, \mathbb{R}^{2}\right)}^{2}
$$

by using (4.2b), (4.2c), (4.2f), Lemma 4.3 (ii), (iii), and Lemma 4.4. Furthermore,

$$
\int_{M_{d^{*} / 2}}\left|D_{3}\right|^{2} \leqslant c\left(l_{*}^{-1}, l^{*},\left\|g_{t}\right\|_{C^{1,0}\left(\overline{\mathbb{S}}_{T}, \mathbb{R}^{2}\right)}\right) \int_{M_{d^{*} / 2}}|D \mathcal{G} \circ \mathcal{H}-D \mathcal{G}|^{2} .
$$

For $\alpha \in[0,1]$ define the auxiliary mapping $\mathcal{H}^{\alpha}: \mathbb{R} \times \mathbb{S} \times\left[0, t^{*}\right] \rightarrow \mathbb{R} \times \mathbb{S}$ by

$$
\mathcal{H}^{\alpha}(d, u, t):=\left(d+\alpha\left(\mathcal{H}_{1}(d, u, t)-d\right), e^{i \alpha \phi} u\right) \quad \text { with } \quad \phi=\arg \left(\frac{\mathcal{H}_{2}(d, u, t)}{u}\right),
$$


where $\left(\mathcal{H}_{1}, \mathcal{H}_{2}\right):=\mathcal{H}$. Observing that $\mathcal{H}^{0}(\cdot, t)$ is the identity on $\mathbb{R} \times \mathbb{S}, \mathcal{H}^{1}=\mathcal{H},\left|\partial_{\alpha} \mathcal{H}^{\alpha}(d, u, t)\right|=$ $d_{\mathbb{R} \times \mathbb{S}}(\mathcal{H}(d, u, t),(d, u))$, and that $\mathcal{H}^{\alpha}$ is bilipschitz with similar estimates like $\mathcal{H}$, we deduce

$$
\int_{M_{d^{*} / 2}}|D \mathcal{G} \circ \mathcal{H}-D \mathcal{G}|^{2} \leqslant 4 \sup _{\mathbb{R} \times \mathbb{S}} d_{\mathbb{R} \times \mathbb{S}}(\mathcal{H}(\cdot, t), \cdot)^{2} \int_{M_{d^{*} / 2}}\left|\partial_{d} D \mathcal{G}\right|^{2}+\left|\partial_{u} D \mathcal{G}\right|^{2} .
$$

This, (4.2g), (4.2h), $d^{*} \leqslant 1$, Lemma 4.3 (i) and the embedding $H^{1}\left(\mathbb{S}, \mathbb{R}^{2}\right) \hookrightarrow C^{0}\left(\mathbb{S}, \mathbb{R}^{2}\right)$ yield

$$
\int_{M_{d^{*} / 2}}\left|D_{3}\right|^{2} \leqslant c\left(1+\left\|g_{\text {иии }}\right\|_{L_{2}\left(\mathbb{S}, \mathbb{R}^{2}\right)}^{2}\right)\|G-g\|_{H^{1}\left(\mathbb{S}, \mathbb{R}^{2}\right)}^{2}
$$

with $c=c\left(\mathrm{DQ}_{*}^{-1},\left\|g_{t}\right\|_{C^{0}\left(\overline{\mathbb{S}}_{T}, \mathbb{R}^{2}\right)},\left\|g_{u}\right\|_{C^{1}\left(\overline{\mathbb{S}}_{T}, \mathbb{R}^{2}\right)}\right)$. In a similar way we arrive at

$$
\int_{M_{d^{*} / 2}}\left|D_{4}\right|^{2} \leqslant c\left(\mathrm{DQ}_{*}^{-1},\left\|g_{u}\right\|_{C^{1}\left(\overline{\mathbb{S}}_{T}, \mathbb{R}^{2}\right)}\right)\left(1+\left\|g_{t u u}\right\|_{L_{2}\left(\mathbb{S}, \mathbb{R}^{2}\right)}^{2}\right)\|G-g\|_{H^{1}\left(\mathbb{S}, \mathbb{R}^{2}\right)}^{2}
$$

and the estimate of $\left\|\left(H^{-1}\right)_{t}\right\|_{L_{2}(\Omega)}$ is established.

LEMMA 4.6 Suppose that (1+) in Lemma 4.1,(2+) in Definition 4.1, and

$$
\|G-g\|_{W_{\infty}^{1}\left(\mathbb{S}, \mathbb{R}^{2}\right)} \leqslant \frac{\mathrm{DQ}_{*}}{8\|n\|_{C^{1,0}\left(\overline{\mathbb{S}}_{T}, \mathbb{R}^{2}\right)}} \quad \text { in }\left[0, t^{*}\right]
$$

hold. Then, for any $t \in\left[0, t^{*}\right]$ the boundary of Int $(G(t))$ is locally a Lipschitz graph. Moreover, we have $(\varphi \circ H)(g)=\varphi(G)$ and

$$
\|\varphi(G)\|_{L_{2}(\mathbb{S})}^{2} \leqslant c\left(\varepsilon^{1 / 2}\|\nabla \varphi\|_{L_{2}(\Omega)}^{2}+\varepsilon^{-1 / 2}\|\varphi\|_{L_{2}(\Omega)}^{2}\right)
$$

for any $\varepsilon \in(0,1], \varphi \in H^{1}(\Omega)$, and $t \in\left[0, t^{*}\right]$. Here $c$ depends on $L_{*}^{-1}, l_{*}^{-1}, l^{*},\left(d^{*}\right)^{-1}$, and $\kappa^{*}$ increasingly.

Proof. At first let us sketch the proof that the boundary of Int $(G(t))$ is Lipschitz. Let $u_{0} \in \mathbb{S}$. We use coordinates given by $\tau_{0}:=g_{u}\left(\sigma\left(u_{0}, t\right), t\right) /\left|g_{u}\left(\sigma\left(u_{0}, t\right), t\right)\right|$ and $n_{0}:=n\left(\sigma\left(u_{0}, t\right), t\right)$. Let us write $G\left(\exp (i r) u_{0}, t\right)=G\left(u_{0}, t\right)+\alpha_{1}(r) \tau_{0}+\alpha_{2}(r) n_{0}, r \in \mathbb{R}$. Check that Assumption (5+) guarantees $\alpha_{1}^{\prime}(r) \geqslant L_{*} / 4>0$ for small $|r|$. So, $\partial \operatorname{Int}(G(t))$ can be represented close to $G\left(u_{0}, t\right)$ as the graph of a composition of $\alpha_{2}$ and a local inverse of $\alpha_{1}$. This composition is Lipschitz continuous, as $\alpha_{1}^{\prime}(r) \geqslant L_{*} / 4$ for small $|r|$ and $G(t) \in W_{\infty}^{1}\left(\mathbb{S}, \mathbb{R}^{2}\right)$.

Consequently, the expression $\varphi(G(t)):=\left.\varphi\right|_{\partial \operatorname{Int}(G(t))} \circ G(t)$ is well defined for any $\varphi \in H^{1}(\Omega)$ and $t \in\left[0, t^{*}\right]$. Furthermore, the identity $(\varphi \circ H)(g)=\varphi(G)$ follows from $H(g)=G$ and an approximation of $\varphi$ by smooth functions.

We still have to show the estimate of $\|\varphi(G)\|_{L_{2}(\Omega)}$. Without loss of generality we may assume that $N$ is the outer normal of $\operatorname{Int}(G)$. Apply [16, Theorem 1.5.1.10] with the vector field

$$
v(x):= \begin{cases}\eta(\operatorname{sdist}(g(t), x)) \partial_{d} \mathcal{G}\left(\mathcal{G}^{-1}(x, t), t\right), & \text { if } x \in U_{d^{*}}(g(\mathbb{S}, t)) \\ 0, & \text { otherwise. }\end{cases}
$$

This vector field satisfies $\|v\|_{C^{1}\left(\bar{\Omega}, \mathbb{R}^{2}\right)} \leqslant c\left(l_{*}^{-1}, l^{*},\left(d^{*}\right)^{-1}, \kappa^{*}\right)$ and, due to $(5+)$, the inequality $N \cdot v(G) \geqslant \frac{1}{2} l_{*}$. Combining all this with Assumption (1+), we get the claimed estimate. 
The identity $G_{t} \cdot G_{u}^{\perp}-g_{t} \cdot g_{u}^{\perp}=\left(G_{t}-g_{t}\right) \cdot G_{u}^{\perp}+g_{t} \cdot\left(G_{u}^{\perp}-g_{u}^{\perp}\right)$ and Lemma 4.6 yield

$$
\begin{aligned}
|\mathcal{I}| \leqslant \varepsilon \int_{0}^{t} \int_{\mathbb{S}}\left|G_{t}-g_{t}\right|^{2}\left|G_{u}\right|^{2}+\varepsilon & \left\|g_{t}\right\|_{C^{0}\left(\overline{\mathbb{S}}_{T}, \mathbb{R}^{2}\right)}^{2} \int_{0}^{t}\left\|G_{u}-g_{u}\right\|_{L_{2}\left(\mathbb{S}, \mathbb{R}^{2}\right)}^{2} \\
& +\varepsilon \int_{0}^{t}\|\nabla(\Theta-\Lambda \hat{\theta})\|_{L_{2}(\Omega)}^{2}+\frac{c}{\varepsilon^{3}} \int_{0}^{t}\|\Theta-\Lambda \hat{\theta}\|_{L_{2}(\Omega)}^{2}
\end{aligned}
$$

for small $\varepsilon>0$. Inserting this inequality with a sufficiently small $\varepsilon$ and Lemma 4.5 in (4.12) we arrive at

$$
\begin{aligned}
\frac{1}{2}\|\Theta(t)-\Lambda \hat{\theta}(t)\|_{L_{2}(\Omega)}^{2}+\frac{1}{2} \int_{0}^{t} & \|\nabla(\Theta-\Lambda \hat{\theta})\|^{2} \leqslant \frac{1}{2} \int_{0}^{t}\left\|G_{t}-g_{t}\right\|_{L_{2}\left(\mathbb{S}, \mathbb{R}^{2}\right)}^{2} \\
& +c \int_{0}^{t} \mathcal{A}^{2}\|G-g\|_{H^{1}\left(\mathbb{S}, \mathbb{R}^{2}\right)}^{2}+c \int_{0}^{t}\|\Theta-\Lambda \hat{\theta}\|_{L_{2}(\Omega)}^{2},
\end{aligned}
$$

provided assumptions $(1+)-(5+)$ hold. Here $c$ depends on the quantities $L_{*}^{-1}, \mathrm{DQ}_{*}^{-1}, \operatorname{dist}_{*}^{-1}$, $\left\|g_{u}\right\|_{C^{1}\left(\overline{\mathbb{S}}_{T}, \mathbb{R}^{2}\right)},\left\|g_{t}\right\|_{C^{0}\left(\overline{\mathbb{S}}_{T}, \mathbb{R}^{2}\right)},\left\|\nabla \theta, \theta_{t}\right\|_{L_{\infty}(Q)}$ increasingly and $\mathcal{A}$ is defined as in Lemma 4.5.

As a preliminary of the estimates for $\Lambda \hat{\theta}-\hat{\theta}$, let us prove the following lemma. Set

$$
M:=\sup _{t \in(0, T)}\left(\left\|\left.\theta\right|_{Q^{s}}(t)\right\|_{H^{2}\left(\omega^{s}(t)\right)}+\left\|\left.\theta\right|_{Q^{l}}(t)\right\|_{H^{2}\left(\omega^{l}(t)\right)}\right)
$$

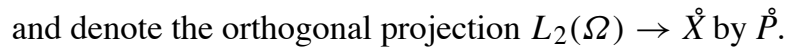

Lemma 4.7 Suppose that (2+)-(4+) and $h \in(0,1]$ hold. Then, we have

$$
\|\dot{P} \hat{\theta}(t)-\hat{\theta}(t)\|_{L_{2}(\Omega)} \leqslant c_{1} h
$$

and

$$
\begin{aligned}
& \int_{0}^{t}\|\dot{P} \hat{\theta}-\hat{\theta}\|_{L_{2}(\Omega)}^{2}, h^{2} \int_{0}^{t}\|\nabla(\grave{P} \hat{\theta}-\hat{\theta})\|_{L_{2}(\Omega)}^{2} \\
& \leqslant c_{2} h^{3}\left(1+\int_{0}^{t}\left(1+\left\|g_{\text {иии }}\right\|_{L_{2}\left(\mathbb{S}, \mathbb{R}^{2}\right)}^{2}\right) h^{-2}\|G-g\|_{H^{1}\left(\mathbb{S}, \mathbb{R}^{2}\right)}^{2}\right)
\end{aligned}
$$

for any $t \in\left[0, t^{*}\right]$. Here $c_{1}$ depends on $l_{*}^{-1}, l^{*},\|\theta\|_{W_{\infty}^{1}(Q)}, c_{\text {aprx }}$ increasingly and on $\Omega$; the constant $c_{2}$ additionally depends on $\mathrm{DQ}_{*}^{-1}, \operatorname{dist}_{*}^{-1},\left\|g_{u}\right\|_{C^{1,0}\left(\mathbb{S}_{T}, \mathbb{R}^{2}\right)}, M, c_{\text {inv }}$, and $T$.

Proof. Let us prove the estimate with $c_{1}$ first. For any $t \in\left[0, t^{*}\right]$ we have

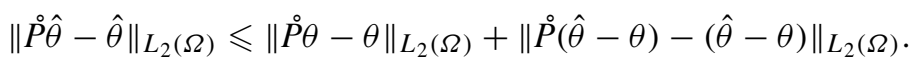

Assumptions (3.5) and (3.6) imply the inequalities

$$
\begin{aligned}
\|\stackrel{\circ}{\theta}-\theta\|_{L_{2}(\Omega)} & \leqslant c_{\text {aprx }} h\|\theta\|_{H^{1}(\Omega)}, \\
\|\stackrel{\circ}{P}(\hat{\theta}-\theta)-(\hat{\theta}-\theta)\|_{L_{2}(\Omega)} & \leqslant c_{\text {aprx }} h\|\hat{\theta}-\theta\|_{H^{1}(\Omega)} .
\end{aligned}
$$


Since

$$
\begin{array}{rlrl}
\left\|\varphi \circ H^{-1}-\varphi\right\|_{L_{2}(\Omega)} \leqslant c\left(l_{*}^{-1}, l^{*}\right)\|\varphi\|_{L_{2}(\Omega)} & & \forall \varphi \in L_{2}(\Omega), \\
\left\|\nabla\left(\varphi \circ H^{-1}-\varphi\right)\right\|_{L_{2}(\Omega)} \leqslant c\left(l_{*}^{-1}, l^{*}\right)\|\nabla \varphi\|_{L_{2}(\Omega)} & \forall \varphi \in H^{1}(\Omega),
\end{array}
$$

and $\hat{\theta} \in C^{0}\left(\left[0, t^{*}\right], L_{2}(\Omega)\right)$, the estimate with $c_{1}$ is established.

In order to prove the estimates with $c_{2}$ we rely on (4.15), (4.16),

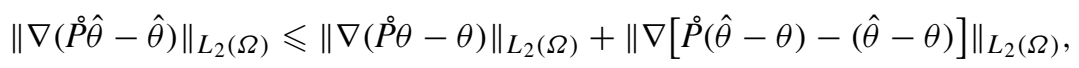

and

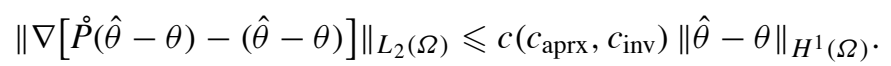

Besides, we use the theory of interpolation spaces (see for example [3] or [4]). The real interpolation space of the Banach spaces $A$ and $B$ with 'order' $(\alpha, q) \in(0,1) \times[1, \infty]$ is denoted by $[A, B]_{\alpha, q}$. Modifying the proof of [24, Theorem 1] slightly we check that the Assumptions (3.8) imply $\theta(t) \in$ $\left[H^{1}(\Omega), H^{2}(\Omega)\right]_{1 / 2, \infty}$ with

$$
\|\theta(t)\|_{\left[H^{1}(\Omega), H^{2}(\Omega)\right]_{1 / 2, \infty}} \leqslant c\left(\left(d^{*}\right)^{-1}, l^{*},\|\theta\|_{W_{\infty}^{1}(Q)}, M, \Omega\right)
$$

for almost every $t \in[0, T]$. An application of the Interpolation Lemma (e.g. [4, (12.1.5)]) on (3.5) with $m=1,2$ yields

$$
\|I \varphi-\varphi\|_{L_{2}(\Omega)}+h\|\nabla(I \varphi-\varphi)\|_{L_{2}(\Omega)} \leqslant c_{\mathrm{aprx}} h^{3 / 2}\|\varphi\|_{\left[H^{1}(\Omega), H^{2}(\Omega)\right]_{1 / 2, \infty}}
$$

for any $\varphi \in\left[H^{1}(\Omega), H^{2}(\Omega)\right]_{1 / 2, \infty}$. Hence,

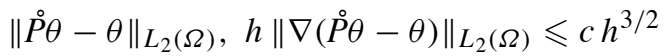

in $[0, T]$, where $c$ depends on $\left(d^{*}\right)^{-1}, l^{*},\|\theta\|_{W_{\infty}^{1}(Q)}, M, c_{\text {aprx }}, c_{\text {inv }}$ increasingly and on $\Omega$. We still have to estimate $\|\hat{\theta}-\theta\|_{H^{1}(\Omega)}$ in such way that we take advantage of the $\left[H^{1}(\Omega), H^{2}(\Omega)\right]_{1 / 2, \infty}$ regularity of $\theta$. We have (compare (4.13))

$$
\begin{aligned}
&\|\varphi \circ H-\varphi\|_{L_{2}(\Omega)} \leqslant c\left(l_{*}^{-1}, l^{*}\right)\|\varphi \circ \mathcal{G} \circ \mathcal{H}-\varphi \circ \mathcal{G}\|_{L_{2}\left(M_{d^{*} / 2}\right)} \\
& \leqslant c\left(\mathrm{DQ}_{*}^{-1}, l^{*}\right)\|\nabla \varphi\|_{L_{2}(\Omega)}\|G-g\|_{H^{1}\left(\mathbb{S}, \mathbb{R}^{2}\right)}
\end{aligned}
$$

for any $\varphi \in H^{1}(\Omega)$. Together with $\|\psi\|_{L_{2}(\Omega)} \leqslant c\left(l_{*}^{-1}, l^{*}\right)\|\psi \circ H\|_{L_{2}(\Omega)}$ this yields

$$
\left\|\varphi \circ H^{-1}-\varphi\right\|_{L_{2}(\Omega)} \leqslant c\left(\mathrm{DQ}_{*}^{-1}, l^{*}\right)\|\nabla \varphi\|_{L_{2}(\Omega)}\|G-g\|_{H^{1}\left(\mathbb{S}, \mathbb{R}^{2}\right)}
$$

for any $\varphi \in H^{1}(\Omega)$. Hence,

$$
\|\hat{\theta}-\theta\|_{L_{2}(\Omega)} \leqslant c\left(\mathrm{DQ}_{*}^{-1}, l^{*},\|\theta\|_{W_{\infty}^{1}(Q)}, \Omega\right)\|G-g\|_{H^{1}\left(\mathbb{S}, \mathbb{R}^{2}\right)}
$$

in $\left[0, t^{*}\right]$. Interpolation of (4.17) and (4.20) gives

$$
\left\|\varphi \circ H^{-1}-\varphi\right\|_{L_{2}(\Omega)} \leqslant c\left(\mathrm{DQ}_{*}^{-1}, l^{*}\right)\|G-g\|_{H^{1}\left(\mathbb{S}, \mathbb{R}^{2}\right)}^{1 / 2}\|\varphi\|_{\left[L_{2}(\Omega), H^{1}(\Omega)\right]_{1 / 2, \infty}}
$$


for any $\varphi \in\left[L_{2}(\Omega), H^{1}(\Omega)\right]_{1 / 2, \infty}$. Therefore, with the help of

$$
\|\nabla \varphi\|_{\left[L_{2}(\Omega), H^{1}(\Omega)\right]_{1 / 2, \infty}} \leqslant\|\varphi\|_{\left[H^{1}(\Omega), H^{2}(\Omega)\right]_{1 / 2, \infty}}
$$

for any $\varphi \in\left[H^{1}(\Omega), H^{2}(\Omega)\right]_{1 / 2, \infty}$ and

$$
\left\|\nabla\left(\varphi \circ H^{-1}-\varphi\right)\right\|_{L_{2}(\Omega)} \leqslant\|\nabla \varphi\|_{L_{\infty}(\Omega)}\left\|D\left(H^{-1}\right)-E\right\|_{L_{2}(\Omega)}+\left\|(\nabla \varphi) \circ H^{-1}-\nabla \varphi\right\|_{L_{2}(\Omega)}
$$

for any $\varphi \in W_{\infty}^{1}(\Omega)$, we arrive at (compare Lemma 4.5)

$$
\begin{aligned}
& \|\nabla(\hat{\theta}-\theta)\|_{L_{2}(\Omega)} \\
& \leqslant c\left(\left(1+\left\|g_{\text {uиu }}\right\|_{L_{2}\left(\mathbb{S}, \mathbb{R}^{2}\right)}^{2}\right)^{1 / 2}\|G-g\|_{H^{1}\left(\mathbb{S}, \mathbb{R}^{2}\right)}+\|G-g\|_{H^{1}\left(\mathbb{S}, \mathbb{R}^{2}\right)}^{1 / 2}\right)
\end{aligned}
$$

and thus

$$
\begin{aligned}
\|\hat{\theta}-\theta\|_{H^{1}(\Omega)} \leqslant c\left(\left(1+\left\|g_{\text {uии }}\right\|_{L_{2}\left(\mathbb{S}, \mathbb{R}^{2}\right)}^{2}\right)^{1 / 2}\|G-g\|_{H^{1}\left(\mathbb{S}, \mathbb{R}^{2}\right)}+\|G-g\|_{H^{1}\left(\mathbb{S}, \mathbb{R}^{2}\right)}^{1 / 2}\right) \\
\underset{h \leqslant 1}{\leqslant} c h^{1 / 2}\left(1+\left(1+\left\|g_{\text {иии }}\right\|_{L_{2}\left(\mathbb{S}, \mathbb{R}^{2}\right)}^{2}\right)^{1 / 2} h^{-1}\|G-g\|_{H^{1}\left(\mathbb{S}, \mathbb{R}^{2}\right)}\right)
\end{aligned}
$$

with $c$ depending on $\mathrm{DQ}_{*}^{-1}$, dist ${ }_{*}^{-1},\left\|g_{u}\right\|_{C^{1,0}\left(\overline{\mathbb{S}}_{T}, \mathbb{R}^{2}\right)},\|\theta\|_{W_{\infty}^{1}(Q)}, M$ increasingly and on $\Omega$. Collecting all the above estimates, we get

$$
\begin{aligned}
& \|\hat{P} \hat{\theta}-\hat{\theta}\|_{L_{2}(\Omega)}, h\|\nabla(\dot{P} \hat{\theta}-\hat{\theta})\|_{L_{2}(\Omega)} \\
& \leqslant c h^{3 / 2}\left(1+\left(1+\left\|g_{\text {uuu }}\right\|_{L_{2}\left(\mathbb{S}, \mathbb{R}^{2}\right)}^{2}\right)^{1 / 2} h^{-1}\|G-g\|_{H^{1}\left(\mathbb{S}, \mathbb{R}^{2}\right)}\right) .
\end{aligned}
$$

We square these inequalities, integrate in time, and arrive at the claimed ones with $c_{2}$.

Lemma 4.8 Suppose that (2+)-(4+) and $h \in(0,1]$ hold. Then,

$$
\begin{aligned}
\|(\Lambda \hat{\theta})(t)-\hat{\theta}(t)\|_{L_{2}(\Omega)}^{2}+\int_{0}^{t} & \|\nabla(\Lambda \hat{\theta}-\hat{\theta})\|_{L_{2}(\Omega)}^{2} \\
& \leqslant c_{1} h\left(1+\int_{0}^{t}\left(1+\left\|g_{\text {uиu }}\right\|_{L_{2}\left(\mathbb{S}, \mathbb{R}^{2}\right)}^{2}\right) h^{-2}\|G-g\|_{H^{1}\left(\mathbb{S}, \mathbb{R}^{2}\right)}^{2}\right)
\end{aligned}
$$

and

$$
\int_{0}^{t}\|\Lambda \hat{\theta}-\hat{\theta}\|_{L_{2}(\Omega)}^{2} \leqslant c_{2} h^{3}\left(1+\int_{0}^{t} \mathcal{A}^{2} h^{-2}\|G-g\|_{H^{1}\left(\mathbb{S}, \mathbb{R}^{2}\right)}^{2}+h \int_{0}^{t}\left\|G_{t}-g_{t}\right\|_{L_{2}\left(\mathbb{S}, \mathbb{R}^{2}\right)}^{2}\right)
$$

for any $t \in\left[0, t^{*}\right]$. Here $c_{1}$ depends on $\mathrm{DQ}_{*}^{-1}, \operatorname{dist}_{*}^{-1},\left\|g_{u}\right\|_{C^{1,0}\left(\overline{\mathbb{S}}_{T}, \mathbb{R}^{2}\right)},\|\theta\|_{W_{\infty}^{1}(Q)}, M, c_{\text {aprx }}, c_{\text {inv }}, T$ increasingly and on $\Omega$; the constant $c_{2}$ depends on $\left\|g_{t}\right\|_{C^{1,0}\left(\overline{\mathbb{S}}_{T}, \mathbb{R}^{2}\right)}$ and $c_{\text {ell }}$ additionally. The function $\mathcal{A}$ is defined as in Lemma 4.5.

Proof. In order to prove the estimate with $c_{1}$ follow the lines of [28, Theorem 1.3], but replace $R \hat{\theta}$ by $\dot{P} \hat{\theta}$ and use the previous Lemma 4.7. Then the estimate with $c_{2}$ follows by a standard duality argument, which resorts to Assumption (3.1), Equality (4.11), and the estimate of $\left\|\left(H^{-1}\right)_{t}\right\|_{L_{2}(\Omega)}$ (see the proof of Lemma 4.5). 
Control of the free boundary (Part 2) and coupling

In Lemma 4.1 the error of the free boundary is controlled in terms of the error of the interfacial temperature. In order to couple this inequality with (4.14) we still have to estimate the error of the interfacial temperature. With the help of Lemma 4.6, we observe that it can be controlled by the part $\Theta-\hat{\theta}$ of the error of the temperature field. More precisely, we have

$$
\begin{aligned}
\|\Theta(G)-\theta(g)\|_{L_{2}(\mathbb{S})}^{2} & =\|(\Theta-\hat{\theta})(G)\|_{L_{2}(\mathbb{S})}^{2} \\
\leqslant & 2\left(\|(\Theta-\Lambda \hat{\theta})(G)\|_{L_{2}(\mathbb{S})}^{2}+\|(\Lambda \hat{\theta}-\hat{\theta})(G)\|_{L_{2}(\mathbb{S})}^{2}\right) \\
& \leqslant \varepsilon\|\nabla(\Theta-\Lambda \hat{\theta})\|_{L_{2}(\Omega)}^{2}+\frac{c}{\varepsilon}\|\Theta-\Lambda \hat{\theta}\|_{L_{2}(\Omega)}^{2} \\
& +c h\|\nabla(\Lambda \hat{\theta}-\hat{\theta})\|_{L_{2}(\Omega)}^{2}+\frac{c}{h}\|\Lambda \hat{\theta}-\hat{\theta}\|_{L_{2}(\Omega)}^{2}
\end{aligned}
$$

for any $h \in(0,1]$ and small $\varepsilon>0$, provided assumptions (1+), $(2+)$, and (5+) hold. We integrate in time, assume that we can apply Lemma 4.8 and get for any $t \in\left[0, t^{*}\right]$

$$
\begin{aligned}
\int_{0}^{t} \| \Theta(G) & -\theta(g)\left\|_{L_{2}(\mathbb{S})}^{2} \leqslant \varepsilon \int_{0}^{t}\right\| \nabla(\Theta-\Lambda \hat{\theta})\left\|_{L_{2}(\Omega)}^{2}+\frac{c_{1}}{\varepsilon} \int_{0}^{t}\right\| \Theta-\Lambda \hat{\theta} \|_{L_{2}(\Omega)}^{2} \\
& +c_{2} h^{2}\left(1+\int_{0}^{t} \mathcal{A}^{2} h^{-2}\|G-g\|_{H^{1}\left(\mathbb{S}, \mathbb{R}^{2}\right)}^{2}\right)+c_{3} h^{3} \int_{0}^{t}\left\|G_{t}-g_{t}\right\|_{L_{2}\left(\mathbb{S}, \mathbb{R}^{2}\right)}^{2},
\end{aligned}
$$

where $c_{1}, c_{2}$, and $c_{3}$ depend on the constants in Lemma 4.6 and Lemma 4.8.

In the following proposition we finish the first stage of the proof by summarizing the results that we have obtained so far.

Proposition 4.1 Let $t^{*} \in[0, T]$ and suppose that $(\Theta, G)$ is a solution on $\left[0, t^{*}\right]$ of the semidiscrete Problem 2.2, which satisfies $G \in C^{1}\left(\left[0, t^{*}\right], Y\right)$ and assumptions (1+)-(5+). Moreover, suppose that

$$
h \leqslant \bar{h}:=\min \left\{1,\left(\frac{1}{4 c c_{3}}\right)^{1 / 3}\right\},
$$

where $c$ is as in Lemma 4.1 and $c_{3}$ as in (4.23). Then, for any $t \in\left[0, t^{*}\right]$

$$
\begin{aligned}
& \frac{1}{4} \int_{0}^{t}\|\nabla(\Theta-\Lambda \hat{\theta})\|_{L_{2}(\Omega)}^{2}+\frac{1}{4} \int_{0}^{t}\left\|G_{t}-g_{t}\right\|_{L_{2}\left(\mathbb{S}, \mathbb{R}^{2}\right)}^{2} \\
& +\frac{1}{2}\|\Theta(t)-(\Lambda \hat{\theta})(t)\|_{L_{2}(\Omega)}^{2}+\|G(t)-g(t)\|_{H^{1}\left(\mathbb{S}, \mathbb{R}^{2}\right)}^{2} \\
& \quad \leqslant c_{1}\left(\int_{0}^{t}\|\Theta-\Lambda \hat{\theta}\|_{L_{2}(\Omega)}^{2}+\int_{0}^{t} \mathcal{A}^{2}\|G-g\|_{H^{1}\left(\mathbb{S}, \mathbb{R}^{2}\right)}^{2}+h^{2}\right)
\end{aligned}
$$

Here the function $\mathcal{A}$ is defined as in Lemma 4.5 and the constant $c_{1}$ depends on the quantities $L_{*}^{-1}$, $L^{*}, \mathrm{DQ}_{*}^{-1}, \operatorname{dist}_{*}^{-1},\|g\|_{C^{2,1}\left(\overline{\mathbb{S}}_{T}, \mathbb{R}^{2}\right)},\left\|g_{t u}\right\|_{C^{0}\left(\overline{\mathbb{S}}_{T}, \mathbb{R}^{2}\right)}, \int_{0}^{T}\left\|g_{t}\right\|_{H^{2}\left(\mathbb{S}_{\mathbb{R}} \mathbb{R}^{2}\right)}^{2},\|\theta\|_{W_{\infty}^{1}(Q)}, M, c_{\mathrm{aprx}}, c_{\mathrm{inv}}, c_{\mathrm{ell}}$, $T$ increasingly and on $\beta, a, \Omega$. 
Furthermore, there exists a constant $c_{2}$, depending on $\int_{0}^{T}\left\|g_{u}\right\|_{H^{2}\left(\mathbb{S}, \mathbb{R}^{2}\right)}^{2}$ additionally, such that

$$
h\|\Theta-\theta\|_{L_{2}(\Omega)}^{2}+\|G-g\|_{H^{1}\left(\mathbb{S}, \mathbb{R}^{2}\right)}^{2} \leqslant c_{2} h^{2} \quad \text { in }\left[0, t^{*}\right] .
$$

Proof. Inequality (4.25) follows from Lemma 4.1, inequalities (4.14), (4.23), and Assumption (4.24). Applying Gronwall's Lemma to this inequality we get

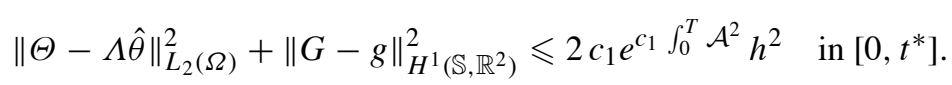

Using this in Lemma 4.8 and (4.21) we estimate

$$
h\|(\Lambda \hat{\theta})(t)-\hat{\theta}(t)\|_{L_{2}(\Omega)}^{2},\|\hat{\theta}(t)-\theta(t)\|_{L_{2}(\Omega)}^{2} \leqslant c\left(c_{1}, \int_{0}^{T} \mathcal{A}^{2}\right) h^{2} .
$$

for all $t \in\left[0, t^{*}\right]$. Thus, the second estimate is established too.

Now, we turn to the second stage of the proof, namely that we establish Inequality (4.25) on $[0, T]$ for small $h$ without supposing the additional assumptions $(1+)-(5+)$. This crucial step relies on the estimate with $c_{2}$ in the previous Proposition 4.1 and the inverse estimate (3.4).

Lemma 4.9 There is a $h^{*}>0$ such that for all $h \in\left(0, h^{*}\right]$ there exists a unique semi-discrete solution $(\Theta, G)$ on $[0, T]$ of Problem 2.2 with $G \in C^{1}([0, T], Y)$. Moreover, $(\Theta, G)$ satisfies Inequality (4.25) for all $t \in[0, T]$. The constant $h^{*}$ depends on the same quantities as $c_{2}$ in Proposition 4.1, but in a decreasing manner.

Proof. For the sake of simplicity, we prove only a simplified claim by assuming the (unique) existence of a solution $(\Theta, G)$ of Problem 2.2 with $G \in C^{1}([0, T], Y)$. The proof of the general claim is obtained along the same lines; one just modifies the following definition of the set $\mathcal{C}_{h}$ and uses the local existence result in Lemma 2.1.

Set $K:=\sqrt{2 c_{2}}$, where $c_{2}$ is as in Proposition 4.1. Let us consider the set

$$
\mathcal{C}_{h}:=\left\{t^{*} \in[0, T] \mid \forall t \in\left[0, t^{*}\right] h\|\Theta(t)-\theta(t)\|_{L_{2}(\Omega)}^{2}+\|G(t)-g(t)\|_{H^{1}\left(\mathbb{S}, \mathbb{R}^{2}\right)}^{2}<K^{2} h^{2}\right\} .
$$

Without loss of generality we can assume that $K$ is so large that the approximation of the initial data implies $\mathcal{C}_{h} \neq \emptyset$. Besides, $\mathcal{C}_{h}$ is open by definition. So, since $[0, T]$ is connected, we can prove $\mathcal{C}_{h}=[0, T]$ by verifying the implication

$$
\left[0, t^{*}\right) \subset \mathcal{C}_{h} \Longrightarrow t^{*} \in \mathcal{C}_{h},
$$

which means that $\mathcal{C}_{h}$ is closed. Let $\left[0, t^{*}\right) \subset \mathcal{C}_{h}$. Then we have

$$
h\|\Theta-\theta\|_{L_{2}(\Omega)}^{2}+\|G-g\|_{H^{1}\left(\mathbb{S}, \mathbb{R}^{2}\right)}^{2} \leqslant K^{2} h^{2} \quad \text { in }\left[0, t^{*}\right] .
$$

This, assumptions (3.4), (3.7), and the embedding $H^{1}\left(\mathbb{S}, \mathbb{R}^{2}\right) \hookrightarrow C^{0}\left(\mathbb{S}, \mathbb{R}^{2}\right)$ yield

$$
\|G-g\|_{W_{\infty}^{1}\left(\mathbb{S}, \mathbb{R}^{2}\right)} \leqslant c\left(c_{\text {inv }}, c_{\text {aprx }}, K\right) h^{1 / 2}
$$


for all $h \in(0,1]$. Thus, there exists a $h^{*} \in(0,1]$, such that $h \in\left(0, h^{*}\right]$ implies that assumptions $(2+)-(5+)$ as well as (1+) with $L_{*}=\frac{1}{2} l_{*}$ and $L^{*}=\frac{3}{2} l^{*}$ hold. Without loss of generality we may assume $h^{*} \leqslant \bar{h}$, where $\bar{h}$ is defined as in Proposition 4.1. Consequently, we can apply Proposition 4.1 and conclude

$$
h\left\|\Theta\left(t^{*}\right)-\theta\left(t^{*}\right)\right\|_{L_{2}(\Omega)}^{2}+\left\|G\left(t^{*}\right)-g\left(t^{*}\right)\right\|_{H^{1}\left(\mathbb{S}, \mathbb{R}^{2}\right)}^{2} \leqslant c_{2} h^{2}<K^{2} h^{2},
$$

that is $t^{*} \in \mathcal{C}_{h}$ with the help of the estimate with $c_{2}$ and the definition of $K$. Therefore, $\mathcal{C}_{h}=$ $[0, T]$ and the additional assumptions (as above) are satisfied in $[0, T]$. One further application of Proposition 4.1 thus yields that Inequality (4.25) holds in $[0, T]$.

\section{Error estimates for the unknowns}

Now we are ready to prove Theorem 3.1. Let $h \in\left(0, h^{*}\right]$, where $h^{*}$ is chosen as in the previous Lemma 4.9. Statement (i) of Theorem 3.1 thus is valid and we get

$$
\begin{aligned}
\sup _{(0, T)}\|\Theta-\Lambda \hat{\theta}\|_{L_{2}(\Omega)}^{2}, & \sup _{(0, T)}\|G-g\|_{H^{1}\left(\mathbb{S}, \mathbb{R}^{2}\right)}^{2}, \int_{0}^{T}\|\nabla(\Theta-\Lambda \hat{\theta})\|_{L_{2}(\Omega)}^{2}, \\
& \int_{0}^{T}\left\|G_{t}-g_{t}\right\|_{L_{2}\left(\mathbb{S}, \mathbb{R}^{2}\right)}^{2} \leqslant c\left(c_{1}, \int_{0}^{T} \mathcal{A}^{2}\right) h^{2},
\end{aligned}
$$

by applying Gronwall's Lemma to (4.25). Inserting (4.26) in Inequality (4.23), we obtain the error estimate

$$
\int_{0}^{T}\|\Theta(G)-\theta(g)\|_{L_{2}(\mathbb{S})}^{2} \leqslant c h^{2}
$$

for the interfacial temperature. Here $c$ depends on the same quantities as before. In order to finish the proof, we still have to estimate $\Lambda \hat{\theta}-\theta$ in the corresponding norms. Let us start with the $L_{\infty}\left(L_{2}\right)$ estimate. Although $R\left(\theta_{t}\right)$ and thus $R\left(\hat{\theta}_{t}\right)$ does not exist in general, we have $R \hat{\theta} \in W_{\infty}^{1}(0, T ; \dot{X})$. This can be recognized with the help of $\hat{\theta} \in W_{\infty}^{1}(Q)$ (see (4.11)), integration by parts, and the assumption that elements of $X$ are piecewise smooth on a partition of $\Omega$ into Lipschitz domains. So, using assumption (3.3), we can follow the proof of [28, Theorem 2.6] and obtain

$$
\sup _{(0, T)}\|\Lambda \hat{\theta}-\hat{\theta}\|_{L_{2}(\Omega)} \leqslant c\left(c_{\text {inv }}\right)\left(1+\max \left\{0, \ln \frac{T}{h^{2}}\right\}\right) \sup _{(0, T)}\|R \hat{\theta}-\hat{\theta}\|_{L_{2}(\Omega)} .
$$

Proceeding in a similar manner as in Lemma 4.7, we get

$$
\sup _{(0, T)}\|R \hat{\theta}-\hat{\theta}\|_{L_{2}(\Omega)} \leqslant c h^{3 / 2}
$$

with the help of assumptions (3.1), (3.6) and inequalities (4.19), (4.18). Hence, we have $\sup _{(0, T)}\|\Lambda \hat{\theta}-\hat{\theta}\|_{L_{2}(\Omega)} \leqslant c h$ where $c$ does not depend on $h$. Since

$$
\sup _{(0, T)}\|\hat{\theta}-\theta\|_{L_{2}(\Omega)} \leqslant c h
$$


by inequalities (4.21) and (4.26), the error estimate for $\sup _{(0, T)}\|\Theta-\theta\|_{L_{2}(\Omega)}$ is established. It remains the $L_{2}\left(L_{2}\right)$-estimate for the heat flux. Inserting (4.26) in Lemma 4.8 and (4.22), we obtain

$$
\int_{0}^{T}\|\nabla(\Lambda \hat{\theta}-\hat{\theta})\|_{L_{2}(\Omega)}^{2}, \int_{0}^{T}\|\nabla(\hat{\theta}-\theta)\|_{L_{2}(\Omega)}^{2} \leqslant c h .
$$

Thus, the estimate of the heat flux is established as well and Theorem 3.1 is proved.

\section{Remarks on Optimality}

We first show that the error estimate for the heat flux is of optimal order. The Stefan condition implies a jump of $(\nabla \theta)(t), t \in[0, T]$, across the interface $\gamma(t)$, if the interface is non-stationary. As a consequence, the maximal regularity of $\theta(t)$, measured in $L_{2}(\Omega)$, is $\left[H^{1}(\Omega), H^{2}(\Omega)\right]_{1 / 2, \infty}$ (compare [24]). The interpolation error for $\nabla \theta(t)$ thus is at most of order $1 / 2$ and so the optimal order of the error estimate for the heat flux is established.

Next, we discuss the optimality of the error estimate for the interfacial temperature $\theta(g)$. We rely on the following assumption: in order to estimate the interpolation error for the interfacial temperature $\theta(g)$ one has to apply a trace theorem in $L_{2}$. We thus need at least $1 / 2$-derivative in $L_{2}(\Omega)$ (see [23]) and so the interpolation error for the interfacial temperature is at most of order 1 . Hence, the error estimate of the interfacial temperature is of optimal order.

Since $\theta(g)$ is a coupling quantity, all the other error estimates also are of optimal order, provided the above assumption that one has to use a trace theorem in $L_{2}$.

\section{Error estimates for the phase distribution}

In order to prove Corollary 3.1 we introduce a 'signed Hausdorff distance'

$$
s \delta\left(f_{1}, f_{2}\right):=\sup _{x \in \mathbb{R}^{2}}\left|\operatorname{sdist}\left(f_{1}, x\right)-\operatorname{sdist}\left(f_{2}, x\right)\right|
$$

between two injective parametrizations $f_{1}, f_{2}: \mathbb{S} \rightarrow \mathbb{R}^{2}$. Then the error estimates of Corollary 3.1 are implied by the inequalities

$$
\delta(A \cap C, B \cap C) \leqslant \delta(A, B)
$$

for any non-empty $A, B, C \subset \mathbb{R}^{2}$ with $C \supset \partial A \cup \partial B$ and

$$
s \delta(G(t), g(t)) \leqslant C h
$$

for all $h \in\left(0, h^{*}\right]$ and $t \in[0, T]$.

In order to show the latter we first derive $\operatorname{Or}(G(t))=\operatorname{Or}(g(t))$ for all $t \in[0, T]$. For a fixed $t \in[0, T]$ let us consider

$$
F_{1}(u, \tau):=\mathcal{G}(\tau \rho(u, t), \exp (i \tau \arg (\sigma(u, t) / u)) u, t), \quad u \in \mathbb{S}, \tau \in[0,1] .
$$

In view of (4.5) the mapping $F_{1}$ is a homotopy from $g(t)$ to $G(t)$. Choose $u_{0} \in \mathbb{S}$ and set $x_{0}:=$ $\mathcal{G}\left(-\frac{3}{4} \operatorname{Or}(g(t)) d^{*}, u_{0}, t\right)$. As $x_{0} \in \operatorname{Int}(g(t))$ but $x_{0} \notin F_{1}(\mathbb{S} \times[0,1])$, we arrive at $\operatorname{Or}(G(t))=$ Or $(g(t))$. 
Next, let us verify that

$$
s \delta(G(t), g(t)) \leqslant\|G(t)-g(t)\|_{C^{0}\left(\mathbb{S}, \mathbb{R}^{2}\right)} .
$$

Let $x \in \mathbb{R}^{2}$ and set $r:=\operatorname{sdist}(G(t), x)$ sdist $(g(t), x)$. We consider two cases: $r<0$ and $r \geqslant 0$. In the case $r<0$ let us consider a second homotopy:

$$
F_{2}(u, \tau):=(1-\tau) G(u, t)+\tau g(u, t), \quad u \in \mathbb{S}, \tau \in[0,1] .
$$

From $\operatorname{Or}(G(t))=\operatorname{Or}(g(t))$ we obtain $x \in F_{2}(\mathbb{S} \times[0, T])$ and thus

$$
\begin{aligned}
& |\operatorname{sdist}(G(t), x)-\operatorname{sdist}(g(t), x)| \\
& \qquad=\operatorname{dist}(G(\mathbb{S}, t), x)+\operatorname{dist}(g(\mathbb{S}, t), x) \leqslant\|G(t)-g(t)\|_{C^{0}\left(\mathbb{S}, \mathbb{R}^{2}\right)},
\end{aligned}
$$

that is (4.28). In the easier case $r \geqslant 0$ we get (4.28) by switching to the unsigned distance function dist. So, Inequality (4.28) is established in general and estimate (4.27) follows with the help of the embedding $H^{1}\left(\mathbb{S}, \mathbb{R}^{2}\right) \hookrightarrow C^{0}\left(\mathbb{S}, \mathbb{R}^{2}\right)$ and Theorem 3.1.

\section{Error estimates for the geometric quantities}

Let us prove Corollary 3.2. Since

$$
\begin{gathered}
\|N-n\|_{L_{2}\left(\mathbb{S}, \mathbb{R}^{2}\right)} \leqslant \frac{2}{\mathrm{DQ}_{*}}\left\|G_{u}-g_{u}\right\|_{L_{2}\left(\mathbb{S}, \mathbb{R}^{2}\right)}, \\
\|V-v\|_{L_{2}(\mathbb{S})} \leqslant\left\|G_{t}-g_{t}\right\|_{L_{2}\left(\mathbb{S}, \mathbb{R}^{2}\right)}+\left\|g_{t}\right\|_{C^{0}\left(\overline{\mathbb{S}}_{T}, \mathbb{R}^{2}\right)}\|N-n\|_{L_{2}\left(\mathbb{S}, \mathbb{R}^{2}\right)},
\end{gathered}
$$

and

$$
\begin{aligned}
\|K-k\|_{L_{2}(\mathbb{S})} \leqslant \frac{1}{a_{*}}\left(\|a\|_{W_{\infty}^{1}(\mathbb{S})}\|k\|_{C^{0}\left(\overline{\mathbb{S}}_{T}\right)}+\right. & \left.\|\beta\|_{W_{\infty}^{1}(\mathbb{S})}\|v\|_{C^{0}\left(\overline{\mathbb{S}}_{T}\right)}\right)\|N-n\|_{L_{2}\left(\mathbb{S}, \mathbb{R}^{2}\right)} \\
& +\frac{1}{a_{*}}\|\Theta(G)-\theta(g)\|_{L_{2}(\mathbb{S})}+\frac{\beta^{*}}{a_{*}}\|V-v\|_{L_{2}(\mathbb{S})}
\end{aligned}
$$

for any $t \in[0, T]$, the error estimates in Corollary 3.2 follow from Theorem 3.1.

\section{Error estimates for measuring quantities}

Let us first prove Corollary 3.3 and then Lemma 3.1. We only show the estimate for $V_{\text {tip }}-v_{\text {tip }}$ in Corollary 3.3; the one for $K_{\text {tip }}-k_{\text {tip }}$ can be obtained in the same manner. Suppressing $t$ and writing $\Sigma_{\text {tip }}:=G^{-1} \circ G_{\text {tip }}$, we can estimate

$$
\begin{aligned}
\left|V_{\text {tip }}-v_{\text {tip }}\right| \leqslant\left|V_{\text {tip }}-(Q v)\left(\Sigma_{\text {tip }}\right)\right|+\mid(Q v)\left(\Sigma_{\text {tip }}\right) & -(J v)\left(\Sigma_{\text {tip }}\right) \mid \\
& +\left|(J v)\left(\Sigma_{\text {tip }}\right)-v\left(\Sigma_{\text {tip }}\right)\right|+\left|v\left(\Sigma_{\text {tip }}\right)-v_{\text {tip }}\right| .
\end{aligned}
$$


Moreover, we have

$$
\begin{aligned}
\left|V_{\text {tip }}-(Q v)\left(\Sigma_{\text {tip }}\right)\right| & \leqslant c_{\text {inv }} h^{-1 / 2}\|Q(V-v)\|_{L_{2}(\mathbb{S})} \leqslant c_{\text {inv }} h^{-1 / 2}\|V-v\|_{L_{2}(\mathbb{S})}, \\
\left|(Q v)\left(\Sigma_{\text {tip }}\right)-(J v)\left(\Sigma_{\text {tip }}\right)\right| & \leqslant c_{\text {inv }} h^{-1 / 2}\left(\|Q v-v\|_{L_{2}(\mathbb{S})}+\|J v-v\|_{L_{2}(\mathbb{S})}\right) \\
& \leqslant c\left(c_{\text {inv }}, c_{\text {aprx }}\right) h^{1 / 2}\|v\|_{W_{\infty}^{1}(\mathbb{S})} \\
\left|(J v)\left(\Sigma_{\text {tip }}\right)-v\left(\Sigma_{\text {tip }}\right)\right| & \leqslant c\left(h^{*}, c_{\text {inv }}, c_{\text {aprx }}\right) h^{1 / 2}\|v\|_{W_{\infty}^{1}(\mathbb{S})} \\
\left|v\left(\Sigma_{\text {tip }}\right)-v_{\text {tip }}\right| & \leqslant \frac{\|v\|_{W_{\infty}^{1}(\mathbb{S})}\left|g\left(G^{-1}\left(G_{\text {tip }}\right)\right)-g_{\text {tip }}\right|}{\mathrm{DQ}_{*}} \mid \| \frac{\|v\|_{W_{\infty}^{1}(\mathbb{S})}}{\mathrm{DQ}_{*}}\left(\left|G_{\text {tip }}-g_{\text {tip }}\right|+c\|G-g\|_{H^{1}\left(\mathbb{S}, \mathbb{R}^{2}\right)}\right) .
\end{aligned}
$$

Therefore, using Corollary 3.2, Theorem 3.1 and $\sup _{(0, T)}\|v\|_{W_{\infty}^{1}(\mathbb{S})}<\infty$, we get the claimed estimate for $V_{\text {tip }}-v_{\text {tip }}$.

It remains to prove Lemma 3.1. Clearly, the conditions of Lemma 3.1 determine $G_{\text {tip }}$ uniquely. Next, we show that the set $A:=\left\{x \cdot e \mid x \in \Gamma(t)\right.$ and $\left.x \cdot e^{\perp}=0\right\}$ is non-empty for any $t \in[0, T]$. Using $e_{2} \cdot(D \mathcal{H}(0, u, t)-E) e_{2}=\sigma_{u}(u, t)-1$ and following the lines of Lemma 4.4, on gets that $\sigma(\cdot, t)$ has an inverse, which will be denoted by $\sigma^{-1}(\cdot, t)$. With the abbreviation $\sigma_{\text {tip }}:=g^{-1} \circ g_{\text {tip }}$ we have $g_{u}\left(\sigma_{\text {tip }}(t), t\right) \cdot e=0$ and therefore,

$$
G\left(\sigma^{-1}\left(\sigma_{\text {tip }}(t), t\right), t\right)=g\left(\sigma_{\text {tip }}(t), t\right)+\rho\left(\sigma^{-1}\left(\sigma_{\text {tip }}(t), t\right), t\right) n\left(\sigma_{\text {tip }}(t), t\right) \in A,
$$

which establishes the existence of the mapping $G_{\text {tip }}$. Furthermore, the function $G_{\text {tip }} \cdot e$ is upper semi-continuous and $G_{\text {tip }}$ thus measurable. Finally, we have

$$
\begin{aligned}
G_{\text {tip }}(t) \cdot e-g_{\text {tip }}(t) \cdot e & \leqslant G\left(\Sigma_{\text {tip }}(t), t\right) \cdot e-g\left(\Sigma_{\text {tip }}(t), t\right) \cdot e \\
& \leqslant c\|G(t)-g(t)\|_{H^{1}\left(\mathbb{S}, \mathbb{R}^{2}\right)}
\end{aligned}
$$

and

$$
\begin{aligned}
g_{\text {tip }}(t) \cdot e-G_{\text {tip }}(t) \cdot e & \leqslant g\left(\sigma_{\text {tip }}(t), t\right) \cdot e-G\left(\sigma^{-1}\left(\sigma_{\text {tip }}(t), t\right), t\right) \cdot e \\
& \leqslant\|\rho(\cdot, t)\|_{L_{\infty}(\mathbb{S})} \leqslant c\|G(t)-g(t)\|_{H^{1}\left(\mathbb{S}, \mathbb{R}^{2}\right)}
\end{aligned}
$$

whence $\left|G_{\text {tip }}(t)-g_{\text {tip }}(t)\right| \leqslant c h$ with the help of Theorem 3.1.

\section{Acknowledgements}

Research supported by the project 'Effiziente Simulation und Numerische Analysis der Dynamik von Dendriten', managed by Gerhard Dziuk and Alfred Schmidt, in the DFG Schwerpunkt 'Dynamik: Analysis, effiziente Simulation und Ergodentheorie'. I would like to thank them and Klaus Deckelnick for interesting discussions on the topic of the paper and helpful remarks.

This article is an extract from the author's PhD thesis at the University of Freiburg, Germany. 


\section{REFERENCES}

1. B̈̈NSCH, E. \& SCHMIDT, A. A finite element method for dendritic growth. In TAYLOR, J. E. (ed.), Computational crystal growers workshop. AMS Selected Lectures in Mathematics, pp. 16-20 (1992).

2. Berger, M. S. Nonlinearity and Functional Analysis, Pure and Applied Mathematics. Academic Press, New York (1977).

3. Bergh, J. \& LÖFSTRÖM, J. Interpolation spaces. An Introduction, vol. 223 of Grundlehren der mathematischen Wissenschaften. Springer, New York (1976).

4. BRenner, S. C. \& SCOTT, L. R. The mathematical theory of finite element methods, Vol. 15 of Texts in Applied Mathematics. Springer, New York (1994).

5. BurcKel, R. B. An introduction to classical complex analysis I, Vol. 64 of Lehrbücher und Monographien aus dem Gebiete der exakten Wissenschaften. Mathematische Reihe. Birkhäuser Verlag, Basel (1979).

6. Caginalp, G. Length scales in phase transition models: Phase field, Cahn-Hillard and blow-up problems. In CHADAM, J. et al. (eds), Pattern formation: symmetry methods and applications. Fields Institute for Research in Mathematical Sciences, Waterloo, Canada, American Mathematical Society, Providence, Rhode Island, pp. 67-83 (1996).

7. Chadam, J. \& Caginalp, G. Stability of interfaces with velocity correction term. Rocky Mt. J. Math. 21, (1991) 617-629.

8. Chen, S., Merriman, B. Osher, S., \& Smereka, P. A simple level set method for solving Stefan problems. J. Comput. Phys. (1997).

9. Chen, X. \& Reitich, F. Local existence and uniqueness of solutions of the Stefan problem with surface tension and kinetic undercooling. J. Math. Anal. Applic. 164, (1992) 350-362.

10. Ciarlet, P. G. The finite element methods for elliptic problems, vol. 4 of Studies in Mathematics and its Applications. North-Holland, Amsterdam (1978).

11. Deckelnick, K. \& Dziuk, G. On the approximation of the curve shortening flow. In Bandle, C. et al. (eds), Calculus of variations, applications and computations. Pont-à-Mousson, Longman Scientific \& Technical, pp. 100-108 (1994).

12. Deckelnick, K. ANd Elliott, C. M. Finite element error bounds for curve shrinking with prescribed normal contact to a fixed boundary, Tech. Report 96/20, Centre for Mathematical Analysis and Its Applications, University of Sussex, Falmer, Brighton BN1 9QH, UK, 1996.

13. EIdel'man, S. Parabolic Systems. North-Holland, Amsterdam (1969).

14. Evans, L. C. \& GARIEPY, R. Measure theory and fine properties of functions, Studies in Advanced Mathematics. CRC Press, Boca Raton, Florida, (1992).

15. Glicksman, M. E. \& Marsh, S. P. The dendrite. In Hurle, D. T. J. (ed.), Handbook of crystal growth, vol. 1 North Holland, Amsterdam - London - New York - Tokyo (1993).

16. GRISVARD, P. Elliptic problems in nonsmooth domains, vol. 24 of Monographs and Studies in Mathematics. Pitman Publishing Inc., Massachusetts (1985).

17. Gurtin, M. E. Thermomechanics of evolving phase boundaries in the plane. Clarendon Press, Oxford (1993).

18. Gurtin, M. E. \& Soner, H. M. Some remarks on the Stefan problem with surface structure. Quart. Appl. Math. 52, (1992) 291-303.

19. Juric, D. \& Tryggvason, G. A front-tracking method for dendritic solidification. J. Comput. Phys. 123, (1996) 127-148.

20. LAnger, J. S. Instabilities and pattern formation in crystal growth. Rev. Modern Phys. 52, (1980) 1-28.

21. Nochetto, R. H. \& Verdi, C. Convergence past singularities for a fully discrete approximation of curvature-driven interfaces. SIAM J. Numer. Anal. 34, (1997) 490-512.

22. Schmidt, A. Computation of three dimensional dendrites with finite elements. J. Comput. Phys. 125, 
(1996) 293-312.

23. ScotT, L. R. A sharp form of the Sobolev trace theorem. J. Func. Anal. 25, (1977) 70-80.

24. ScotT, L. R. Applications of Banach space interpolation to finite element theory. In NASHED, M. Z. (ed.), Functional analysis methods in numerical analysis, vol. 701 of Lecture Notes in Mathematics. Springer, New York (1979).

25. SсOTT, L. R. \& ZHANG, S. Finite element interpolation of nonsmooth functions satisfying boundary conditions. Math. Comput. 54, (1990) 483-493.

26. Soner, H. M. Convergence of the phase-field equations to the Mullins-Sekerka problem with kinetic undercooling. Arch. Rational Mech. Anal. 131, (1995) 139-197.

27. Strain, J. Velocity effects in unstable solidification. SIAM J. Appl. Math. 50, (1990) 1-15.

28. Thоме́E, V. Galerkin finite element methods for parabolic problems, vol. 25 of Springer Series in Computational Mathematics. Springer, Berlin (1997).

29. VEESER, A. Fehlerabschätzungen für ein Verfahren zur Berechnung von zweidimensionalen Dendriten, $\mathrm{PhD}$ thesis, Institut für Angewandte Mathematik, Universität Freiburg, April 1998.

30. Visintin, A. Models of phase transitions, vol. 28 of Progress in Nonlinear Differential Equations and Their Applications. Birkhäuser, Boston (1996).

31. WAng, S. L. \& SEKerkA, R. F. Algorithms for phase field computation of the dendritic operating state at large supercoolings. J. Comput. Phys. 127, (1996).

32. ZIEMER, W. P. Weakly differentiable functions, vol. 120 of Graduate Texts in Mathematics. Spinger, New York (1989). 